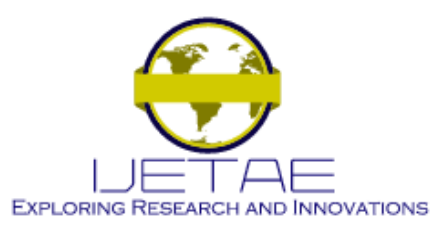

International Journal of Emerging Technology and Advanced Engineering

Website: www.ijetae.com (ISSN 2250-2459, ISO 9001:2008 Certified Journal, Volume 10, Issue 11, November 2020

\title{
MATLAB Simulation for Trajectory/Path Efficiency Comparison Between Robotic Manipulators
}

\author{
Joshua Laber ${ }^{1}$, Ravindra Thamma ${ }^{2}$ \\ ${ }^{1,2}$ Robotics and Mechatronics Engineering Technology, Central Connecticut State University, USA
}

\begin{abstract}
This paper examines results of MATLAB simulation for three robotic arm configurations to determine their abilities in trajectory/path tracing. The trajectories tested were a spline curve, a circle, and square. The joint position vs. time, joint velocity vs. time, joint acceleration vs. time, and the cartesian position vs. time were plotted to compare their agility.
\end{abstract}

Keywords-Robotic Arm, Kinematics, Forward Kinematics, Inverse Kinematics, Velocity Kinematics, Path Planning, Trajectory Generation, MATLAB, Robotics Toolbox, SCARA, Articulated, 5-DoF, 6-DoF, DenavitHartenberg

\section{INTRODUCTION}

Three robotic arms, Fanuc LR Mate 200iC/5H, Fanuc LR Mate 200iD, and Fanuc SR-6iA, were drawn as a set of coordinate frames and their forward kinematics was calculated. The advantages and weaknesses of each robot configuration was compared in their theoretical abilities. This paper validates prior study titled "Efficiency Comparison Between Robotic Manipulator Configurations for Trajectory/Path Tracing" [1]. In that paper it was said that a robot with a lower DoF would be faster than a robot with a higher DoF. Therefore, SCARA would be faster to move from point-to-point than an Articulated Robot and that a robot with lower degree of freedom (DoF) would be faster than one with a higher DoF. The LR Mate $200 \mathrm{iC} / 5 \mathrm{H}$ is a 5-DoF Articulated Arm, the LR Mate 200iD is a 6-DoF Articulated Arm, and the SR-6iA is a 4-DoF SCARA arm.

\section{PRoposed StUdy}

The trajectory planning and path tracing would be simulated in MATLAB using the Robotics Toolbox developed by Peter Corke. For each robot, the joint position vs. time, joint velocity vs. time, joint acceleration vs. time, and the cartesian position vs. time will be plotted. This comparison will further explore the performance of these three arms. The most effective robot would be the one which is able to complete the path in the shortest time.

\section{CAlCulations}

\section{A. Kinematics}

Robot kinematics is the relation of the position of joints and links and the robot's real-world layout. By categorizing a robot into its basic joints and links, the kinematics can be studied. Joints are the connections between rigid links, which constrains the movement between each link to either translation or rotation. Most joints can be described as prismatic or revolute. A prismatic joint allows the links to translate linearly. A revolute joint allows the links to revolve about a point of rotation. The three robot arms can also be defined as serial manipulators, as the joints and links are ordered in a single, non-branching chain. The topology of a robot can be written using alphanumeric notation where " $\mathrm{P}$ " is a prismatic joint and " $\mathrm{R}$ " is a revolute joint from base to end-effector. The notation can be simplified by writing repeated types of types as the number of that type of joint followed by the joint type [2]. The Fanuc LR Mate 200iC/5H is 5R (RRRRR), Fanuc LR Mate 200iD is 6R (RRRRRR), and Fanuc SR-6iA is 2RPR (RRPR). The configuration of the robot describes its position and orientation in space, by defining each degree of freedom. The degrees of freedom (DoF) are defined as the least number of coordinates needed to describe the robot's configuration. C-Space, or configuration space, is the space containing every possible configuration which the robot can attain. The task space is the space which the robot's task is expressed in, i.e. following a given path. The circle and square path are given as real coordinate space $\mathrm{R}^{2}$ about $\mathrm{Y}-\mathrm{Z}$ and $\mathrm{X}-\mathrm{Y}$, respectively, and the spline is expressed as $\mathrm{R}^{3}$. The workspace is the specific set of configurations which the end-effector can reach in C-Space [3]. The C-Space of all three robots is defined as $\mathrm{R}^{3}$, so that all can translate and rotate in $\mathrm{X}-\mathrm{Y}-\mathrm{Z}$ cartesian space. As there are no obstacles, the workspace and C-Space are both equal.

\section{B. Forward Kinematics}

The forward kinematics of a robot is the calculation of the actual coordinates of the joints for a known set of joint parameters. 


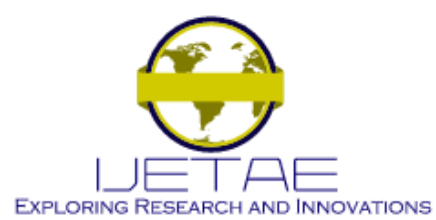

International Journal of Emerging Technology and Advanced Engineering Website: www.ijetae.com (ISSN 2250-2459, ISO 9001:2008 Certified Journal, Volume 10, Issue 11, November 2020

The robot is drawn as a set of coordinate frames so that the kinematics can be calculated. The coordinate frame design used in this paper is described in the paper, Efficiency Comparison Between Robotic Manipulator Configurations for Trajectory/Path Tracing [1]. The paper then describes how the forward kinematics are calculated for each robot by applying the Denavit-Hartenberg (D-H) convention [4]. The D-H convention simplifies each robot into a set of four parameters per joint frame so that positions of the joints can be converted to cartesian position [5]. By calculating the transformation matrices from base to end-effector using the D-H parameters, the cartesian position can be found.

\section{Inverse Kinematics}

Inverse kinematics is the opposite of forward kinematics. It is the calculation of the robot's joint parameters given a cartesian position. Unlike forward kinematics, inverse kinematics can have multiple solutions for the same coordinates [6]. The transformation matrix of the end effector relative to the base is given by Eq.1:

$$
H=\left[\begin{array}{cccc}
r 11 & r 12 & r 13 & o x \\
r 21 & r 22 & r 23 & o y \\
r 31 & r 32 & r 33 & o z \\
0 & 0 & 0 & 1
\end{array}\right] \quad \text { Eq.1[5] }
$$

Where the variables can be solved by calculating the transformation matrix for each joint. The transformation of the end-effector can be found by calculating the product of each transformation matrix $T$ from body to end-effector, such as shown in Eq.2:

$$
{ }_{1}^{0} T_{2}^{1} T{ }_{3}^{2} T \ldots{ }_{n}^{n-1} T={ }_{n}^{0} T \quad \text { Eq.2[7] }
$$

For example, the transformation matrix $H$ for the endeffector frame of the Fanuc 200iD, can be computed as:

$$
\begin{aligned}
& { }_{1}^{0} T=\left[\begin{array}{cccc}
\cos (q 1) & 0 & -\sin (q 1) & 50 * \cos (q 1) \\
\sin (q 1) & 0 & \cos (q 1) & 50 * \sin (q 1) \\
0 & -1 & 0 & 330 \\
0 & 0 & 0 & 1
\end{array}\right] \\
& { }_{2}^{1} T=\left[\begin{array}{cccc}
\cos (q 2) & \sin (q 2) & 0 & 330 * \cos (q 2) \\
\sin (q 2) & -\cos (q 2) & 0 & 330 * \sin (q 2) \\
0 & 0 & -1 & 0 \\
0 & 0 & 0 & 1
\end{array}\right] \quad \text { Eq.4 } \\
& { }_{3}^{2} T=\left[\begin{array}{cccc}
\cos (q 3) & 0 & \sin (q 3) & -35 * \cos (q 3) \\
\sin (q 3) & 0 & -\cos (q 3) & -35 * \sin (q 3) \\
0 & 1 & 0 & 0 \\
0 & 0 & 0 & 1
\end{array}\right]
\end{aligned}
$$

$$
\begin{aligned}
&{ }_{4}^{3} T=\left[\begin{array}{cccc}
\cos (q 4) & 0 & -\sin (q 4) & 0 \\
\sin (q 4) & 0 & \cos (q 4) & 0 \\
0 & -1 & 0 & -335 \\
0 & 0 & 0 & 1
\end{array}\right] \quad \text { Eq.6 } \\
&{ }_{5}^{4} T=\left[\begin{array}{cccc}
\cos (q 5) & 0 & \sin (q 5) & 0 \\
\sin (q 5) & 0 & -\cos (q 5) & 0 \\
0 & 1 & 0 & 0 \\
0 & 0 & 0 & 1
\end{array}\right] \quad \text { Eq.7 } \\
&{ }_{6}^{5} T=\left[\begin{array}{cccc}
\cos (q 6) & \sin (q 6) & 0 & 0 \\
\sin (q 6) & -\cos (q 6) & 0 & 0 \\
0 & 0 & -1 & -80 \\
0 & 0 & 0 & 1
\end{array}\right] \quad \text { Eq.8 } \\
& H={ }_{1}^{0} T_{2}^{1} T_{3}^{2} T_{4}^{3} T_{5}^{4} T_{6}^{5} T \text { Eq. } 9
\end{aligned}
$$

Where $q 1, q 2, q 3, q 4, q 5, q 6$ is the angle of the corresponding joint. Eq.3-8 were derived through the previously calculated D-H parameters of the Fanuc LR Mate 200iD. Eq.9 is Eq.1 restated to fit the question. By computing the product of each frame, the nonlinear trigonometric equations are revealed:

$$
\begin{aligned}
r 11=\cos (q 1) * & (\cos (q 2) *(\cos (q 3) *(\cos (q 4) \\
& * \cos (q 5) * \cos (q 6)-\sin (q 4) \\
& * \sin (q 6))-\sin (q 3) * \sin (q 5) \\
& * \cos (q 6))+\sin (q 2) *(\cos (q 3) \\
& * \sin (q 5) * \cos (q 6)+\sin (q 3) \\
& *(\cos (q 4) * \cos (q 5) * \cos (q 6) \\
& -\sin (q 4) * \sin (q 6)))+\sin (q 1) \\
& *(\cos (q 4) * \sin (q 6)+\sin (q 4) \\
& * \cos (q 5) * \cos (q 6)) \\
r 21=\cos (q 1) * & (-\cos (q 4) * \sin (q 6)-\sin (q 4) \\
& * \cos (q 5) * \cos (q 6))+\sin (q 1) \\
& *(\cos (q 2) *(\cos (q 3) *(\cos (q 4) \\
& * \cos (q 5) * \cos (q 6)-\sin (q 4) \\
& * \sin (q 6))-\sin (q 3) * \sin (q 5) \\
& * \cos (q 6))+\sin (q 2) *(\cos (q 3) \\
& * \sin (q 5) * \cos (q 6)+\sin (q 3) \\
& *(\cos (q 4) * \cos (q 5) * \cos (q 6) \\
& -\sin (q 4) * \sin (q 6)))) \\
r 31=\cos (q 2) * & (\cos (q 3) * \sin (q 5) * \cos (q 6) \\
& +\sin (q 3) *(\cos (q 4) * \cos (q 5) \\
& * \cos (q 6)-\sin (q 4) * \sin (q 6))) \\
& +\sin (q 2) *(\cos (q 3) *(\sin (q 4) \\
& * \sin (q 6)-\cos (q 4) * \cos (q 5) \\
& * \cos (q 6))+\sin (q 3) * \sin (q 5) \\
& * \cos (q 6)), \\
&
\end{aligned}
$$




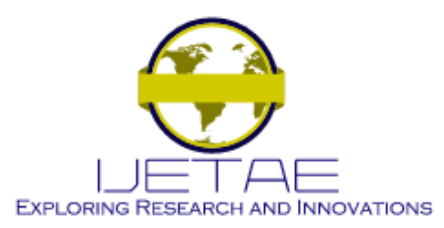

International Journal of Emerging Technology and Advanced Engineering Website: www.ijetae.com (ISSN 2250-2459, ISO 9001:2008 Certified Journal, Volume 10, Issue 11, November 2020

$$
\begin{aligned}
& r 12=\cos (q 1) *(\cos (q 2) *(\cos (q 3) *(\cos (q 4) \\
& * \cos (q 5) * \sin (q 6)+\sin (q 4) \\
& * \cos (q 6))-\sin (q 3) * \sin (q 5) \\
& * \sin (q 6))+\sin (q 2) *(\cos (q 3) \\
& * \sin (q 5) * \sin (q 6)+\sin (q 3) \\
& *(\cos (q 4) * \cos (q 5) * \sin (q 6) \\
& +\sin (q 4) * \cos (q 6))))+\sin (q 1) \\
& *(\sin (q 4) * \cos (q 5) * \sin (q 6) \\
& -\cos (q 4) * \cos (q 6)) \\
& r 22=\cos (q 1) *(\cos (q 4) * \cos (q 6)-\sin (q 4) \\
& * \cos (q 5) * \sin (q 6))+\sin (q 1) \\
& *(\cos (q 2) *(\cos (q 3) *(\cos (q 4) \\
& * \cos (q 5) * \sin (q 6)+\sin (q 4) \\
& * \cos (q 6))-\sin (q 3) * \sin (q 5) \\
& * \sin (q 6))+\sin (q 2) *(\cos (q 3) \\
& * \sin (q 5) * \sin (q 6)+\sin (q 3) \\
& *(\cos (q 4) * \cos (q 5) * \sin (q 6) \\
& +\sin (q 4) * \cos (q 6)))) \\
& \begin{aligned}
r 32=\cos (q 2) * & (\cos (q 3) * \sin (q 5) * \sin (q 6)+\sin (q 3) \\
& *(\cos (q 4) * \cos (q 5) * \sin (q 6) \\
& +\sin (q 4) * \cos (q 6)))+\sin (q 2) \\
& *(\cos (q 3) *(-\cos (q 4) * \cos (q 5) \\
& * \sin (q 6)-\sin (q 4) * \cos (q 6)) \\
& +\sin (q 3) * \sin (q 5) * \sin (q 6))
\end{aligned} \\
& r 13=-(\cos (q 1) *(\cos (q 2) *(\cos (q 3) * \cos (q 4) \\
& * \sin (q 5)+\sin (q 3) * \cos (q 5)) \\
& -\sin (q 2) *(\cos (q 3) * \cos (q 5) \\
& -\sin (q 3) * \cos (q 4) * \sin (q 5))) \\
& +\sin (q 1) * \sin (q 4) * \sin (q 5)) \\
& r 23=\cos (q 1) * \sin (q 4) * \sin (q 5)-\sin (q 1) *(\cos (q 2) \\
& *(\cos (q 3) * \cos (q 4) * \sin (q 5) \\
& +\sin (q 3) * \cos (q 5))-\sin (q 2) \\
& *(\cos (q 3) * \cos (q 5)-\sin (q 3) \\
& * \cos (q 4) * \sin (q 5))) \\
& \begin{aligned}
r 33=\cos (q 2) * & (\cos (q 3) * \cos (q 5)-\sin (q 3) \\
& * \cos (q 4) * \sin (q 5))+\sin (q 2) \\
& *(\cos (q 3) * \cos (q 4) * \sin (q 5) \\
& +\sin (q 3) * \cos (q 5))
\end{aligned} \\
& o x=\cos (q 1) *(\cos (q 2) *(\cos (q 3) *(-80 * \cos (q 4) \\
& * \sin (q 5)-35)+\sin (q 3) *(-80 \\
& * \cos (q 5)-335)+330)+\sin (q 2) \\
& *(\cos (q 3) *(80 * \cos (q 5)+335) \\
& +\sin (q 3) *(-80 * \cos (q 4) * \sin (q 5) \\
& -35))+50)-80 * \sin (q 1) * \sin (q 4) \\
& * \sin (q 5)
\end{aligned}
$$

$$
\begin{aligned}
o y=80 * \cos (q 1) & * \sin (q 4) * \sin (q 5)+\sin (q 1) \\
& *(\cos (q 2) *(\cos (q 3) *(-80 \\
& * \cos (q 4) * \sin (q 5)-35)+\sin (q 3) \\
& *(-80 * \cos (q 5)-335)+330) \\
& +\sin (q 2) *(\cos (q 3) *(80 * \cos (q 5) \\
& +335)+\sin (q 3) *(-80 * \cos (q 4) \\
& * \sin (q 5)-35))+50) \\
o z=\cos (q 2) * & (\cos (q 3) *(80 * \cos (q 5)+335) \\
& +\sin (q 3) *(-80 * \cos (q 4) * \sin (q 5) \\
& -35))+\sin (q 2) *(\cos (q 3) *(80 \\
& * \cos (q 4) * \sin (q 5)+35)+\sin (q 3) \\
& *(80 * \cos (q 5)+335)-330)+330
\end{aligned}
$$

These equations can then be solved to directly calculate the joint position and orientation in closed form [5]. As inverse kinematics can be complicated and tedious to calculate at each point along the path, the calculations were done with Robotics Toolbox for MATLAB.

\section{Inverse Kinematics}

Velocity kinematics are used to calculate the endeffector of the robot's twist given the joint position and velocities. The velocity kinematics can be related by Eq.10:

$$
\dot{x}=J(\theta) \dot{\theta} \quad \text { Eq. } 10[3]
$$

Where $J(\theta)$ is the Jacobian matrix of the position of the joint variables, $\dot{x}$ is the time derivative of the cartesian endeffector velocity, and $\dot{\theta}$ is the time derivative of the joint end-effector velocity. As the inverse kinematics would calculate the joint angles at each point along the path, the velocity can simplify be calculated by taking the derivative of each joint coordinate with respect to time and dividing the result by the sampling rate.

\section{E. Path Planning and Trajectory Generation}

For a robot to smoothly trace a path, the trajectory must be calculated. A path is a sequence of geometric waypoints which the robot achieves along the route were as a trajectory is a path with a time scale, meaning each waypoint has a time when the configuration is accomplished. A trajectory considers constraints such as joint velocities and acceleration. There are a few methods of blending the waypoints of the path into a trajectory: Point-to-point, polynomial via point, and time-optimal time scaling. A straight-line path is a point-to-point path which forms a line from the start position to end position in joint space. As any two points in free space would form a straight-line path passing through free space, all straightline paths are valid. 


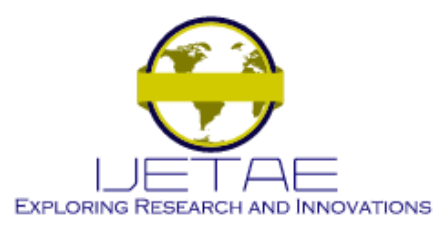

International Journal of Emerging Technology and Advanced Engineering Website: www.ijetae.com (ISSN 2250-2459, ISO 9001:2008 Certified Journal, Volume 10, Issue 11, November 2020

Polynomial via point trajectories use polynomial interpolation to create a trajectory which uses the waypoints as guides without strictly defining the path between. Time-optimal time scaling is mainly used to decrease the time following the path and increase efficiency. As the maximum possible velocity and acceleration change over the course of the path, this planning system would find the most optimal polynomial for that section. [3] For the three robots, the maximum joint velocities and joint angle limits are defined in the datasheets. The type of trajectory planning used in the code is Multi-segment multi-axis trajectory generation. This algorithm generates each a polynomial for each point-topoint segment which the end-effector follows by rotating and translating all the robot's joints simultaneously. [8]

A phase space plot shows the relation of the joint position to the joint velocity. When no point on any of paths operates at a speed greater than the maximum joint velocity and operates at the maximum possible acceleration, the phase plots shows the optimal trajectory curves to complete the path.[9] When a joint does not show movement in the position and velocity space, the joint is an equilibrium point in the phase space.[10] The distance from the origin to the curve is equal to the magnitude of the joint position and the angle of that distance to the polar is proportional to the amount the path is complete.[11]

\section{MATLAB CODE}

\section{A. Robotics Toolbox}

The kinematics of the robots were calculated using Robotics Toolbox for MATLAB[8], version 10.4, by Peter Corke, a professor of robotic vision at Queensland University of Technology.[12] The Robotics Toolbox is a suite of functions that allows for the computation of kinematics, dynamics, and generating trajectories for robotic arm manipulators. The toolbox also includes functions for manipulating vectors and transformations matrices. It is not to be confused with Robotic Systems Toolbox, the toolbox by the MATLAB developer MathWorks.[13]
TABLE I

DESCRIPTION OF PATHS

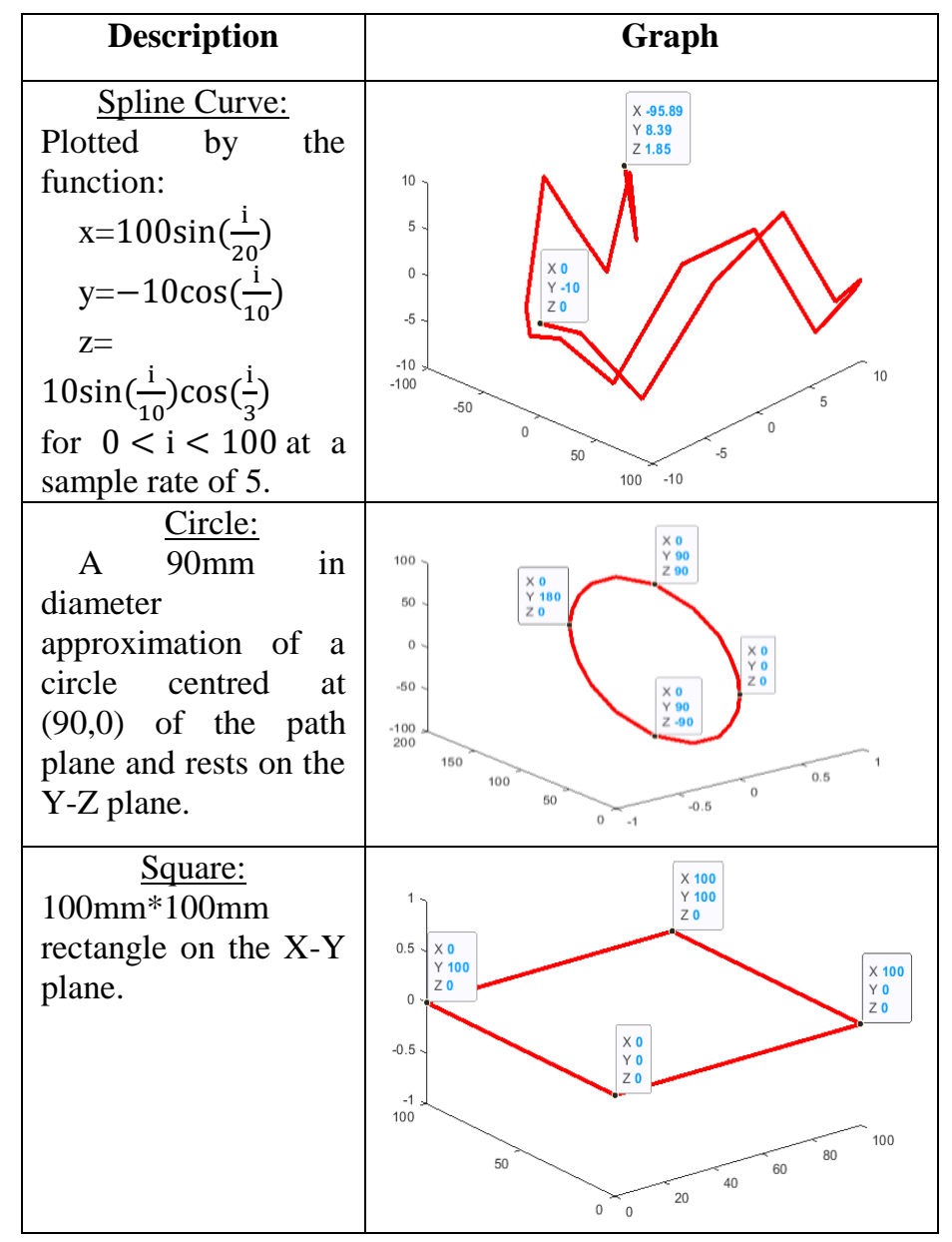

\section{B. Path}

The robots were tested on their agility in following three paths as displayed in the Table I: a spline curve, circle, and square. The spline curve tests the robot's ability to trace an arbitrary curve. The circle tests the robot's ability in following a round object. The square tests the robot's ability to navigate sharp corners. 


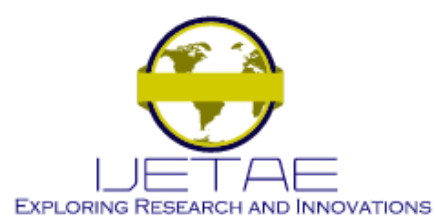

International Journal of Emerging Technology and Advanced Engineering Website: www.ijetae.com (ISSN 2250-2459, ISO 9001:2008 Certified Journal, Volume 10, Issue 11, November 2020

\section{Code Explanation}

The simulation is programmed in MATLAB with the aid of Robotic Toolbox.

Each robot is input as a series of links using their D-H parameters and their joint limits. The links and joint limits for the 200iD are:

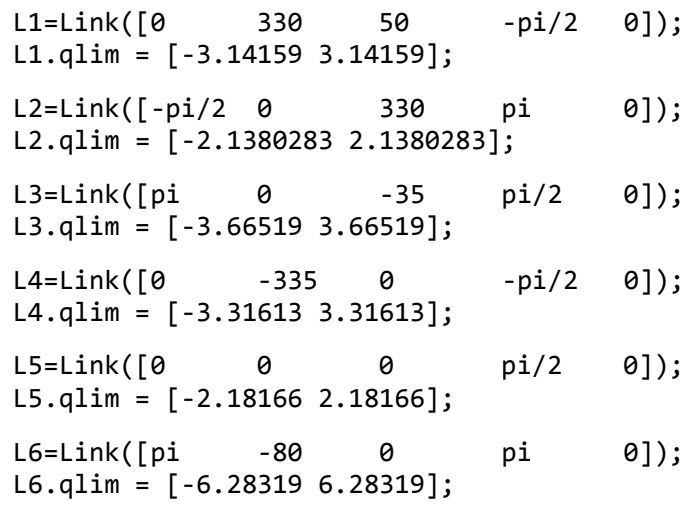

The links are joined collectively as a SerialLink variable: Fan200iD = SerialLink([L1 L2 L3 L4 L5 L6 $]$, 'name', '200iD');

The path is input as a Mx3 matrix, where the rows are the $\mathrm{X}, \mathrm{Y}, \mathrm{Z}$ cartesian coordinates and columns are each separate point on the path as shown in Table II.

TABLE II

Path COORDinates

\begin{tabular}{|c|c|c|c|c|c|}
\hline \multicolumn{3}{|c|}{ Spline } & \multicolumn{2}{|c|}{ Circle } & Square \\
\hline$[0$ & -10 & 0 & {$\left[\begin{array}{lll}0 & 0\end{array}\right.$} & 0 & {$\left[\begin{array}{lll}0 & 0 & 0 ;\end{array}\right.$} \\
\hline 24.74 & -8.78 & -0.46 & 02 & 20 & 1000 \\
\hline 47.94 & -5.40 & -8.26 ; & 010 & 40 & $20 \quad 0 \quad 0$; \\
\hline 68.16 & -0.71 & 2.82 & 022 & 60 & $30 \quad 0 \quad 0$; \\
\hline 84.15 & 4.16 & 8.43 & 049 & 80 & $40 \quad 0 \quad 0$; \\
\hline 94.9 & 8.01 & -2.76 & 090 & 90 & $50 \quad 0 \quad 0 ;$ \\
\hline 99.75 & 9.90 & -1.18; & 0131 & 80 & $60 \quad 0 \quad 0$; \\
\hline 98.4 & 9.36 & -2.18 & 0157 & 60 & $70 \quad 0 \quad 0$; \\
\hline 90.93 & 6.54 & -5.45 & 0170 & 40 & $80 \quad 0 \quad 0$; \\
\hline 77.81 & 2.11 & 7.43; & 0177 & 20 & $90 \quad 0 \quad 0$ \\
\hline 59.85 & -2.84 & 5.51 & 0180 & 0 & $1000 \quad 0$ \\
\hline 38.16 & -7.09 & -6.14 ; & 0177 & -20 ; & 100100 ; \\
\hline 14.11 & -9.60 & -1.14; & 0170 & -40 & 100200 ; \\
\hline-10.82 & -9.77 & -2.04 & 0157 & -60 & 100300 ; \\
\hline-35.08 & -7.54 & -1.49 & 0131 & -80 & 100400 ; \\
\hline-57.16 & -3.47 & 9.3 & 090 & -90 & 100500 ; \\
\hline-75.68 & 1.46 & 0.36 & 049 & -80 & 10060 0; \\
\hline-89.5 & 6.02 & -7.97; & 022 & -60 & 100700 ; \\
\hline-97.75 & 9.11 & 0.64 & 010 & -40 & 100800 ; \\
\hline-99.93 & 9.97 & -7.3 & 02 & -20 & 100900 ; \\
\hline-95.89 & 8.39 & 1.85;]; & 00 & & 100100 0; \\
\hline
\end{tabular}

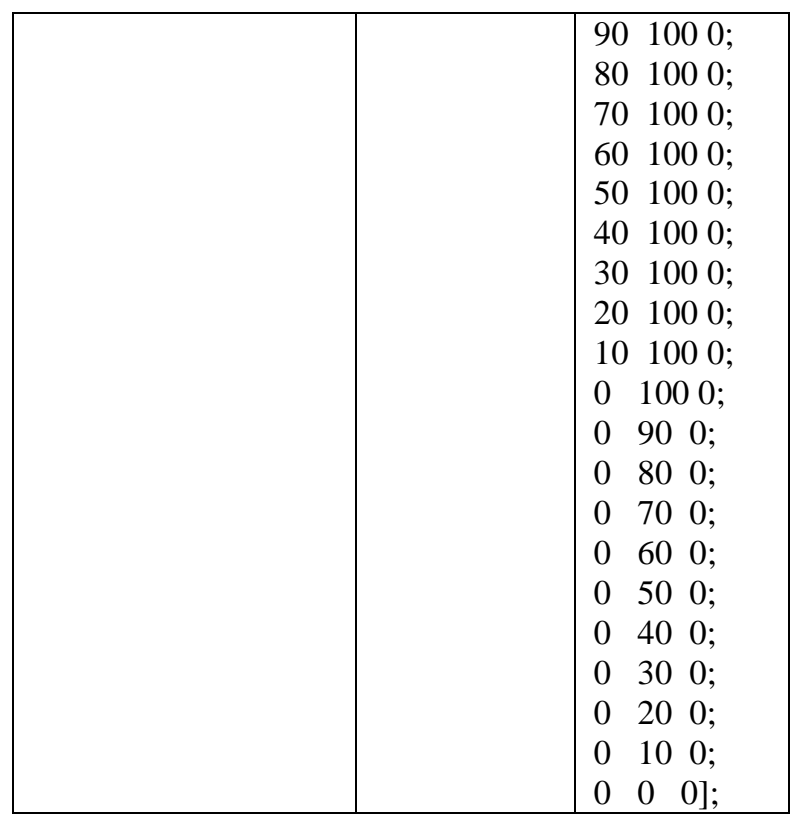

This code allows the path shape to be easily changed by setting three variables that define a path. The path is set to the desired path coordinate matrix. The tacc variable sets the time acceleration which would be used in the trajectory function. The start variable is the starting coordinate which the end-effector would be set to at the beginning of the trajectory.

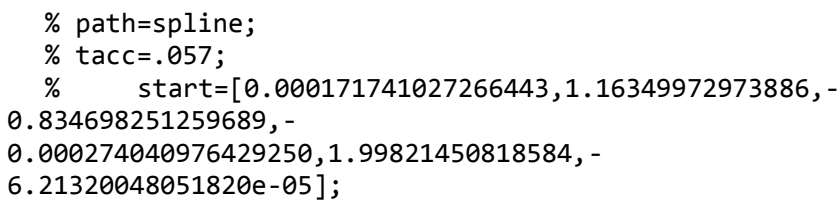

The path is then translated to a location relative to the robotic arm as a set of $4 \times 4$ homogenous matrices. Here the path is placed $500 \mathrm{~mm}$ in the positive $\mathrm{X}$ direction.

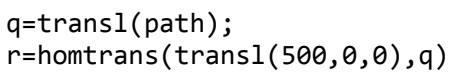

The toolpath, the path the end-effector follows, is displayed as a red line on the 3D plot

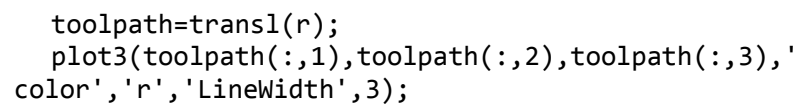

The ikcon function is used to solve the inverse kinematics for the joint angles at the given point, while obeying the joint limits:

$$
\mathrm{S}=\mathrm{Fan200iD} \text {. ikcon }(r) \text {; }
$$




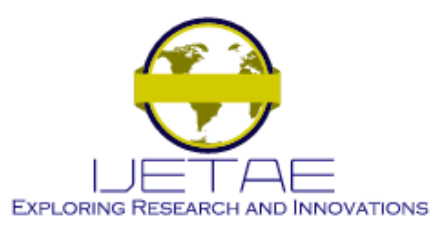

\section{International Journal of Emerging Technology and Advanced Engineering Website: www.ijetae.com (ISSN 2250-2459, ISO 9001:2008 Certified Journal, Volume 10, Issue 11, November 2020}

The ikcon function produces unexpected results when used with the SCARA robot, so instead the ikine function is used. The ikine function solves the inverse kinematics similarly to the ikcon function but does not respect joint limits. Care is provided to prevent the program from producing impossible real-world trajectories.

$$
S=F a n u c S R . i k i n e\left(r, \text { 'mask' , }\left[\begin{array}{llllll}
1 & 1 & 1 & 0 & 0 & 0
\end{array}\right]\right) \text {; }
$$

The trajectory is calculated using the mstraj function. Mstraj, meaning Multi-segment multi-axis trajectory, blends the waypoints of the path together to create a trajectory given the joint angles at each point, the maximum velocity of the points, the initial joint coordinates, time step, and the acceleration time. The initial joint coordinates that were used was the first row of joint path matrix S. By lowering the acceleration time, the speed of the joints of the robot increases. The fastest the robot can perform the task is when a joint reaches its maximum velocity. All calculations were computed with a sampling rate of 0.001 .

limitspeed $=[6.28319 ; 4.27606 ; 7.33038 ; 6.63225 ;$

4.36332; 12.5664];

sampling $=0.001$;

trajectory $=$

mstraj(s, limitspeed, [ ], start, sampling, tacc);

The time the robot took to complete the action is output as the duration.

Totalsteps=size (trajectory, 1$)$ \%total steps

Duration=size (trajectory, 1$)$ *sampling \%duration/cycle time in sec

The trajectory of the velocity, acceleration, and cartesian position are calculated:

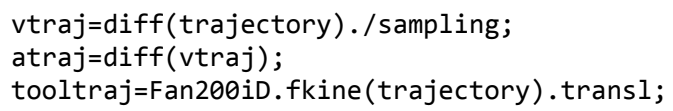

Since the maximum acceleration per joint for the SR-6iA is unknown, the parameters which are used are assumed to be $1 / 5^{\text {th }}$ the joint velocity limits as used by ROS. A function is added in the SR-6iA program to stop the code execution when the robot exceeds the assumed maximum acceleration parameters.

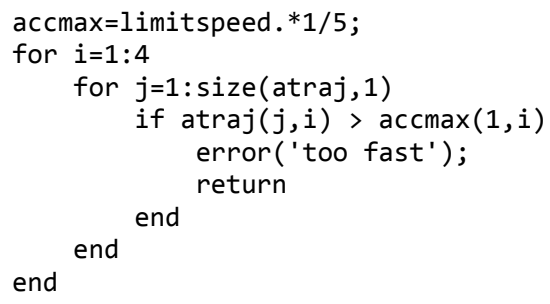

Joint Position vs. Time:

Figure \%joint position vs time graph subplot $(6,1,1)$; plot(trajectory $(:, 1))$; xlabel('Time (s)'); ylabel('Joint 1

(rad) '); xt=get (gca, 'xtick' ); set (gca, 'xticklabel' , array fun(@num2str,xt*sampling, 'un', 0));

subplot $(6,1,2)$; plot(trajectory $(:, 2))$; xlabel('Time (s)'); ylabel('Joint 2

(rad) ') ; xt=get (gca, 'xtick'); set (gca, 'xticklabel' , array fun(@num2str,xt*sampling, 'un', 0));

subplot $(6,1,3)$; plot(trajectory $(:, 3))$; xlabel('Time (s)'); ylabel('Joint 3

(rad) ') ; xt=get (gca, 'xtick'); set (gca, 'xticklabel' , array fun(@num2str,xt*sampling, 'un', 0));

subplot $(6,1,4)$; plot(trajectory $(:, 4))$; xlabel('Time (s)'); ylabel('Joint 4

(rad)' ); xt=get (gca, 'xtick'); set (gca, 'xticklabel ', array fun(@num2str,xt*sampling, 'un' ',0));

subplot $(6,1,5)$; plot(trajectory $(:, 5))$; xlabel('Time (s)'); ylabel('Joint 5

(rad)' ); xt=get (gca, 'xtick'); set(gca, 'xticklabel', array fun(@num2str,xt*sampling, 'un', 0));

subplot $(6,1,6)$; plot(trajectory $(:, 6)$ ); xlabel('Time (s)'); ylabel('Joint 6 (rad)' ); xt=get (gca, 'xtick'); set (gca, 'xticklabel' , array fun(@num2str,xt*sampling, 'un', 0));

\section{Joint Velocity vs. Time:}

figure \%joint velocity vs time graph subplot $(6,1,1)$; plot $(\operatorname{vtraj}(:, 1))$; xlabel('Time (s)'); ylabel('Joint Velocity 1

(rad/s) '); xt=get(gca, 'xtick'); set (gca, 'xticklabel' , arr ayfun(@num2str,xt*sampling, 'un',0));

subplot $(6,1,2)$; plot(vtraj(:,2)); xlabel('Time (s)'); ylabel('Joint Velocity 2

(rad/s)' ); xt=get (gca, 'xtick'); set (gca, 'xticklabel', arr ayfun(@num2str,xt*sampling, 'un',0));

subplot $(6,1,3)$; plot(vtraj(:,3)); xlabel('Time $(\mathrm{s})^{\prime}$ ); ylabel('Joint Velocity 3 (rad/s) ') ; xt=get(gca, 'xtick') ; set (gca, 'xticklabel' , arr ayfun(@num2str,xt*sampling, 'un ',0));

subplot $(6,1,4)$; plot(vtraj(:,4)); xlabel('Time (s)'); ylabel('Joint Velocity 4 (rad/s) '); xt=get(gca, 'xtick' ); set (gca, 'xticklabel' , arr ayfun(@num2str,xt*sampling, 'un',0));

subplot $(6,1,5)$; plot(vtraj(:,5)); xlabel('Time (s)'); ylabel('Joint Velocity 5 (rad/s) '); xt=get(gca, 'xtick'); set (gca, 'xticklabel' , arr ayfun(@num2str,xt*sampling, 'un',0));

subplot $(6,1,6)$; plot(vtraj(:,6)); xlabel('Time (s)'); ylabel('Joint Velocity 6 (rad/s) ') ; xt=get(gca, 'xtick'); set (gca, 'xticklabel ', arr ayfun(@num2str,xt*sampling, 'un',0)); 


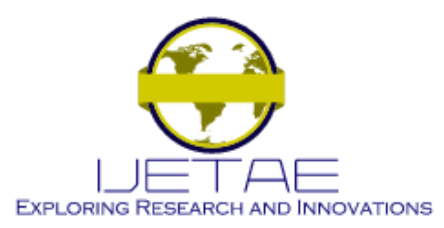

International Journal of Emerging Technology and Advanced Engineering Website: www.ijetae.com (ISSN 2250-2459, ISO 9001:2008 Certified Journal, Volume 10, Issue 11, November 2020

\section{Joint Acceleration vs. Time:}

figure \%joint acceleration vs time graph subplot $(5,1,1)$; plot(atraj $(:, 1))$; xlabel('Time (s)'); ylabel('Joint Acceleration 1 $\left.\left(\mathrm{rad} / \mathrm{s}^{\wedge} 2\right)^{\prime}\right)$; xt=get (gca, 'xtick'); set (gca, 'xticklabel' , a rrayfun(@num2str,xt*sampling, 'un',0));

subplot $(5,1,2)$; plot(atraj $(:, 2)) ; x$ label('Time (s)'); ylabel('Joint Acceleration 2 (rad/s^2) '); xt=get(gca, 'xtick'); set (gca, 'xticklabel', a rrayfun(@num2str,xt*sampling, 'un',0));

subplot $(5,1,3)$; plot(atraj $(:, 3)) ; x$ label('Time (s)'); ylabel('Joint Acceleration 3 $\left.\left(\mathrm{rad} / \mathrm{s}^{\wedge} 2\right)^{\prime}\right)$; xt=get $($ gca, 'xtick'); set (gca, 'xticklabel' , a rrayfun(@num2str,xt*sampling, 'un',0));

subplot $(5,1,4)$; plot(atraj $(:, 4))$; xlabel('Time (s)'); ylabel('Joint Acceleration 4 $\left.\left(\mathrm{rad} / \mathrm{s}^{\wedge} 2\right)^{\prime}\right)$; xt=get (gca, 'xtick'); set (gca, 'xticklabel', a rrayfun(@num2str,xt*sampling, 'un',0));

subplot $(5,1,5)$; plot (atraj $(:, 5)) ; x$ label('Time (s)'); ylabel('Joint Acceleration 5 $\left.\left(\mathrm{rad} / \mathrm{s}^{\wedge} 2\right)^{\prime}\right)$; xt=get (gca, 'xtick'); set (gca, 'xticklabel', a rrayfun(@num2str,xt*sampling, 'un',0));

subplot $(6,1,6)$; plot (atraj $(:, 6))$; xlabel('Time (s)'); ylabel('Joint Acceleration 6 $\left.\left(\mathrm{rad} / \mathrm{s}^{\wedge} 2\right)^{\prime}\right)$; xt=get $($ gca, 'xtick'); set (gca, 'xticklabel' , a rrayfun(@num2str,xt*sampling, 'un',0));

Joint Position vs. Joint Velocity:

figure

subplot $(6,1,1)$; plot (vtraj $(:, 1)$, trajectory $(1:$ end$1,1))$; xlabel('Joint Velocity 1 ( $\mathrm{rad} / \mathrm{s})$ '); ylabel('Joint 1 (rad)'); \%joint position vs joint velocity graph

subplot $(6,1,2)$; plot (vtraj $(:, 2)$, trajectory $(1:$ end$1,2))$; xlabel('Joint Velocity 2 ( $\mathrm{rad} / \mathrm{s})$ '); ylabel('Joint 2 (rad)');

subplot $(6,1,3)$; plot(vtraj $(:, 3)$, trajectory(1:end$1,3))$; xlabel('Joint Velocity 3 ( $\mathrm{rad} / \mathrm{s})$ '); ylabel('Joint 3 (rad)');

subplot $(6,1,4)$; plot (vtraj $(:, 4)$, trajectory $(1:$ end$1,4))$; xlabel('Joint Velocity 4 ( $\mathrm{rad} / \mathrm{s})$ '); ylabel('Joint 4 (rad)');

subplot $(6,1,5)$; plot (vtraj $(:, 5)$, trajectory(1:end$1,5))$; xlabel('Joint Velocity 5 ( $\mathrm{rad} / \mathrm{s})$ '); ylabel('Joint 5 (rad)');

subplot $(6,1,6)$; plot (vtraj $(:, 6)$, trajectory $(1:$ end$1,6))$; xlabel('Joint Velocity 6 ( $\mathrm{rad} / \mathrm{s})$ '); ylabel('Joint 6 (rad)');

Cartesian Position vs. Time:

subplot $(3,1,1)$; plot(tooltraj(:,1)); xlabel('Time (s)'); ylabel('X (mm)');

xt=get (gca, 'xtick'); set (gca, 'xticklabel' , arrayfun(@num 2str, $x t^{*}$ sampling, 'un',0)); subplot $(3,1,2)$; plot(tooltraj(:,2)); xlabel('Time (s)'); ylabel('Y (mm)'); xt=get (gca, 'xtick'); set (gca, 'xticklabel', arrayfun(@num 2str, xt*sampling, 'un',0));

subplot $(3,1,3)$; plot(tooltraj(:,3)); xlabel('Time (s)'); ylabel('z (mm)'); xt=get (gca, 'xtick'); set (gca, 'xticklabel' , arrayfun(@num 2str, $x^{*}$ sampling, 'un',0));

The graphs are calculated and plotted for each axis. The plots for the joint position, velocity, and acceleration have a subplot for each joint. So, on the Fanuc LR Mate 200iD, there are 6 subplots each, on the $200 \mathrm{iC} / 5 \mathrm{H}$ there are 5 subplots, and on the SR-6iA there are 4 subplots. Each revolute joint movement is measured in radians and the prismatic joint is measured in $\mathrm{mm}$. The $\mathrm{x}$ axis for each subplot is remapped from samples to seconds by multiplying the sample by the sampling rate, which is given in samples per second.

\section{RESUlTS}

The performance of the robots is compared by their time to complete the three paths. Each trajectory is defined by the path coordinates matrix, the starting position, and the acceleration time. The starting position is the coordinates of the joints at the beginning of the path, which is in cartesian terms: $0,-10,0$ for the spline path and $0,0,0$ for the circle and square paths. The acceleration time is chosen by lowering the value until the robot is unable to complete the path without breaking its set maximum joint velocity. The program outputs the time duration and steps, as well as the trajectory animation, and multiple plots which describe the trajectory.

TABLE III

Spline Path Results

\begin{tabular}{|l|l|l|l|}
\hline & 200iD & 200iC/5H & SR-6iA \\
\hline Tacc & 0.057 & 0.0230 & 0.009 \\
\hline Steps & 2374 & 946 & 370 \\
\hline Duration (s) & 2.3740 & 0.9460 & 0.3700 \\
\hline
\end{tabular}

According to Table III, the SR-6iA completed the spline path $2.56 \mathrm{x}$ faster than the second quickest time of the 200iC/5H. The SR-6iA also had the lowest acceleration time. The $200 \mathrm{iC} / 5 \mathrm{H}$ was second and the $200 \mathrm{iD}$ was third.

TABLE IV Circle Path Results

\begin{tabular}{|l|l|l|l|}
\hline & 200iD & 200iC & SR-6iA \\
\hline Tacc & 0.0301 & 0.0140 & 0.014 \\
\hline Steps & 1282 & 588 & 588 \\
\hline Duration (s) & 1.2820 & 0.58800 & 0.5880 \\
\hline
\end{tabular}




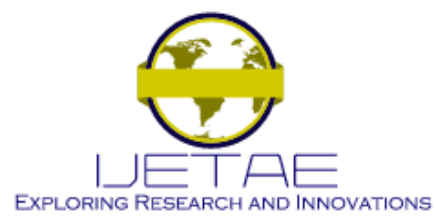

International Journal of Emerging Technology and Advanced Engineering Website: www.ijetae.com (ISSN 2250-2459, ISO 9001:2008 Certified Journal, Volume 10, Issue 11, November 2020

In Table IV, the 200iC and SR-6iA tied for lowest acceleration time at 0.014 , step count at 588 , and time duration at 0.588 seconds. The $200 \mathrm{iD}$ was $2.18 \mathrm{x}$ slower than the two other robots.

TABLE V

SQUARE PATH RESUlts

\begin{tabular}{|l|l|l|l|}
\hline & 200iD & 200iC & SR-6iA \\
\hline Tacc & 0.0251 & 0.0131 & 0.007 \\
\hline Steps & 2132 & 1148 & 534 \\
\hline Duration (s) & 2.1320 & 1.1480 & 0.5340 \\
\hline
\end{tabular}

In Table V, the SR-6iA was quickest and 2.18x faster and had at $1.87 \mathrm{x}$ lower acceleration time than the $200 \mathrm{iC}$. The $200 \mathrm{iC} / 5 \mathrm{H}$ was $1.86 \mathrm{x}$ faster than the $200 \mathrm{iD}$ and had a $1.92 x$ lower acceleration time.

TABLE VI SPline Path Plots

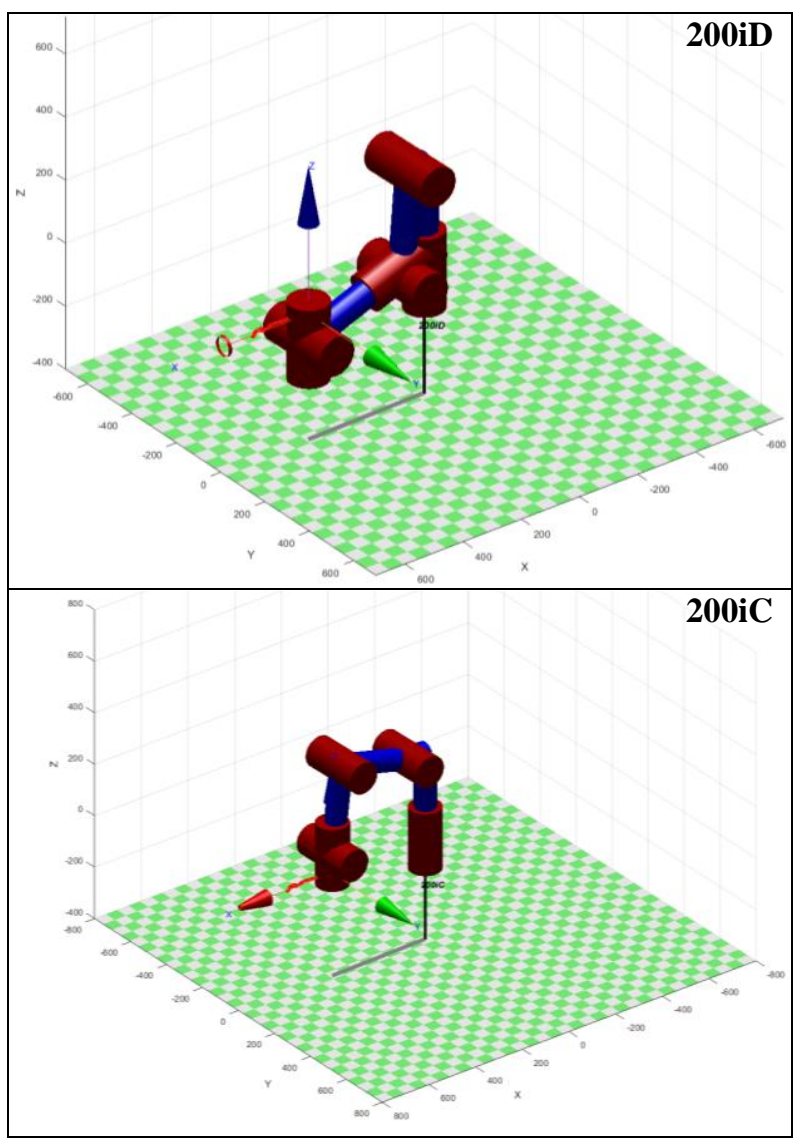

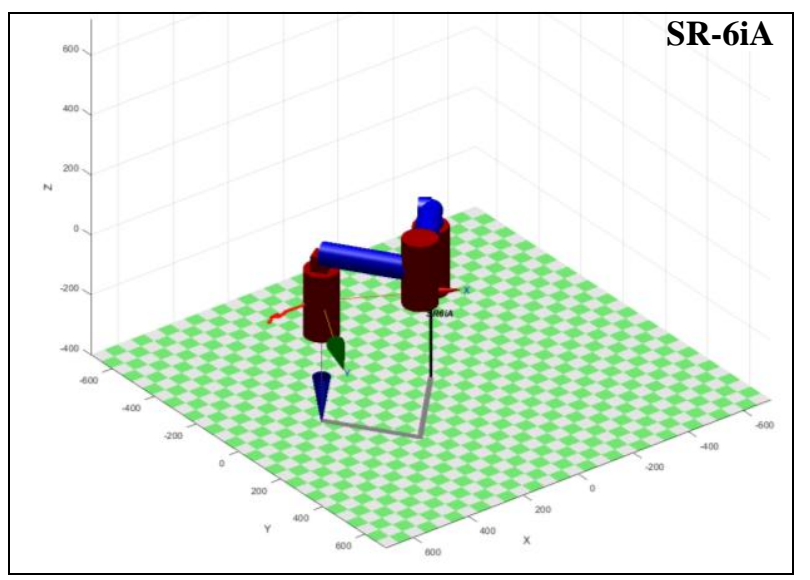

The 3D plots in Table VI show the configuration of the 3 robots follow the spline trajectory. The 200iD is following with the end-effector oriented upwards, while the $200 \mathrm{iC}$ and SR-6iA have downward oriented end-effectors.

TABLE VII Circle Path Plots

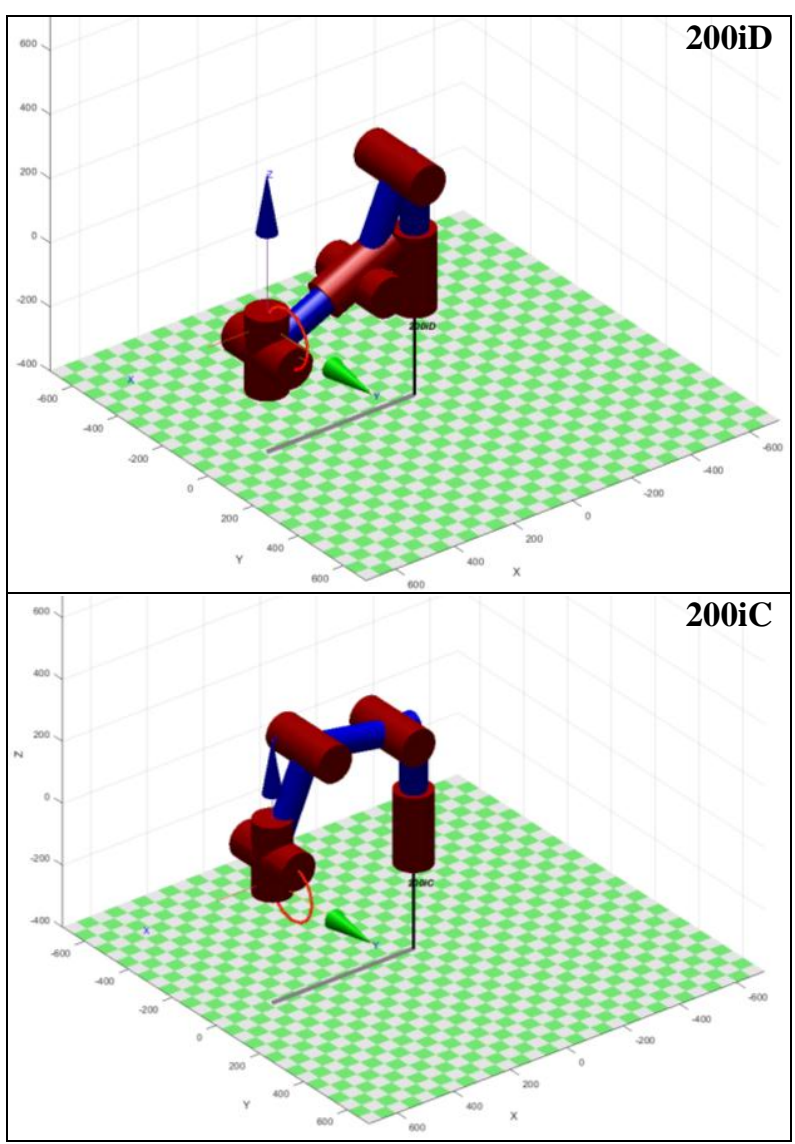




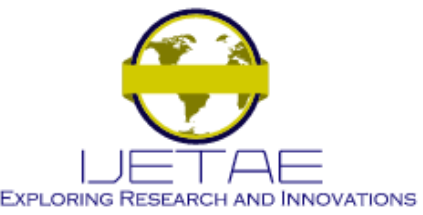

International Journal of Emerging Technology and Advanced Engineering Website: www.ijetae.com (ISSN 2250-2459, ISO 9001:2008 Certified Journal, Volume 10, Issue 11, November 2020

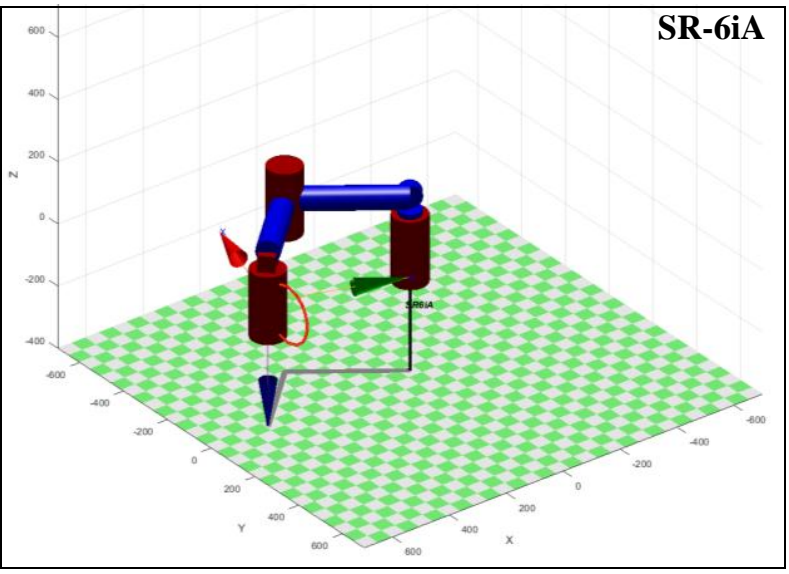

The 3D plots in Table VII show the configuration of the 3 robots follow the circle trajectory. The 200iD has an upward oriented end-effector, while the 200iC and SR-6iA have downward oriented end-effectors.

TABLE VIII SQUARE PATH Plots

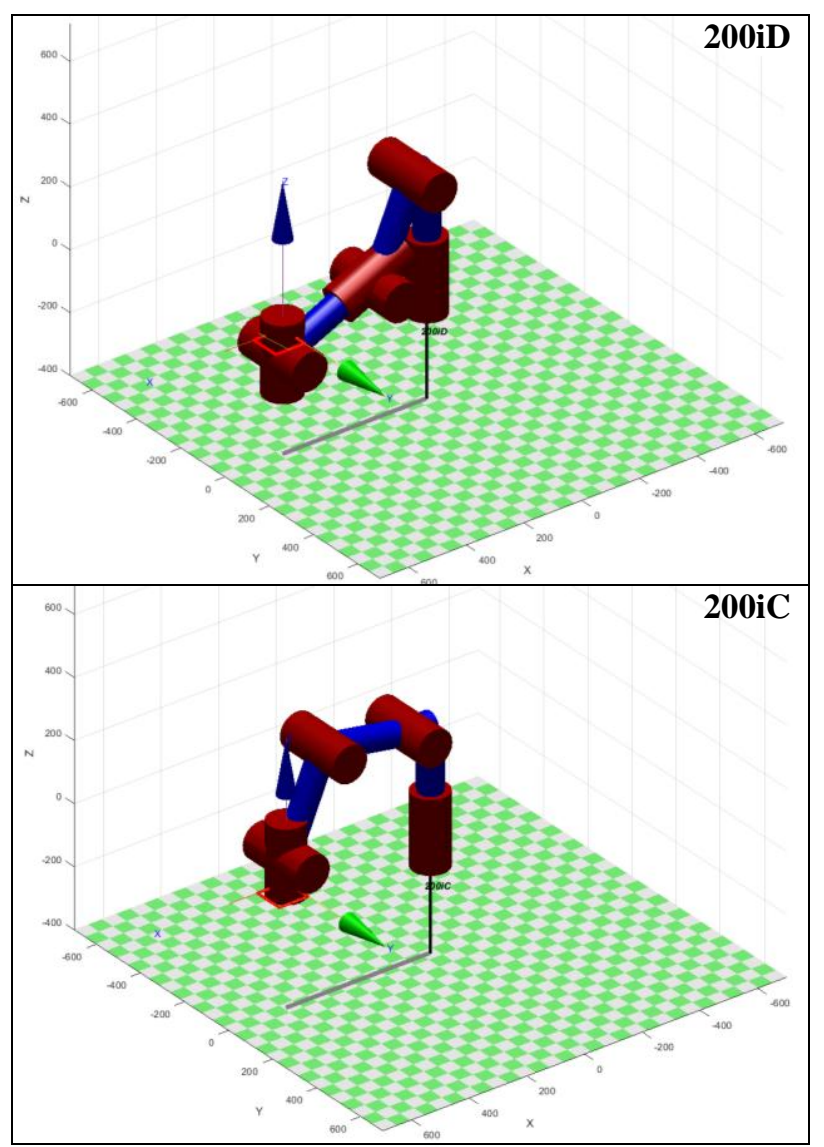

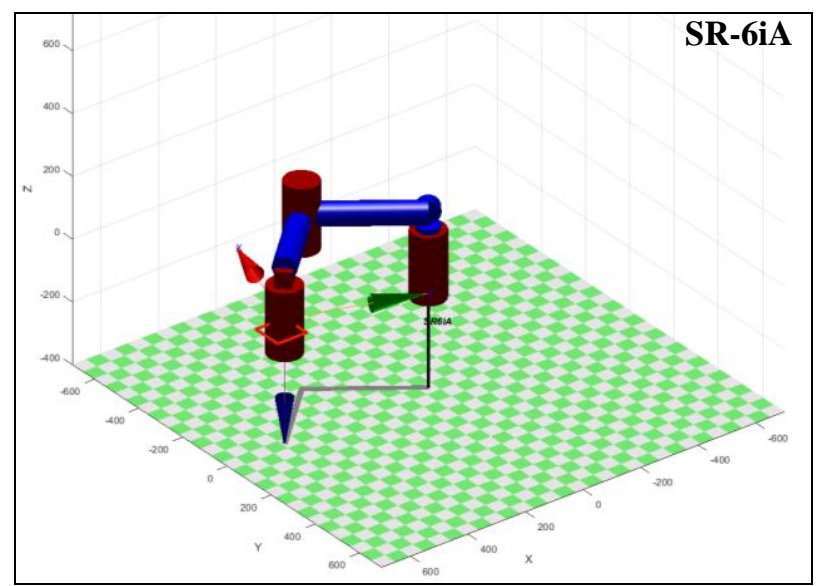

This 3D plots in Table VIII show the configuration of the 3 robots follow the square trajectory. The 200iD has an upward oriented end-effector, while the 200iC and SR-6iA have downward oriented end-effectors.

TABLE IX

Spline Path Joint Position vs. Time

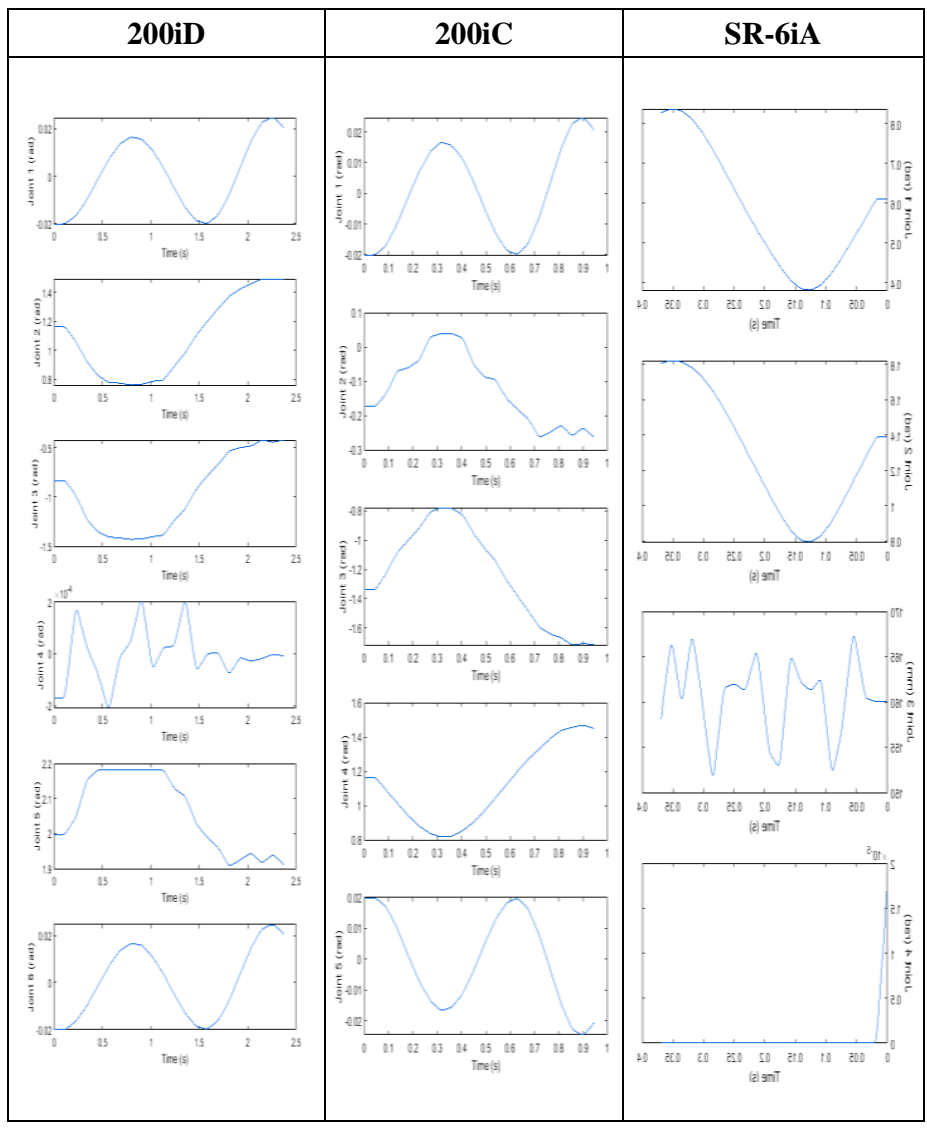




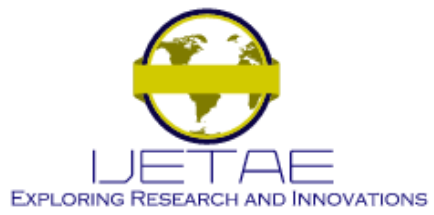

International Journal of Emerging Technology and Advanced Engineering Website: www.ijetae.com (ISSN 2250-2459, ISO 9001:2008 Certified Journal, Volume 10, Issue 11, November 2020

Table IX shows the joint position vs. time of the spline graphs for the three robots. Joint position is the angle of the joint relative to the robots' zero position. The time is measured in seconds. The maximum value of the 200iD is $2.1817 \mathrm{rad}$ on joint 5 and minimum is $-1.4305 \mathrm{rad}$ on joint 3. The maximum value of the $200 \mathrm{iC}$ is $1.468 \mathrm{rad}$ on joint 4 and minimum is $-1.7143 \mathrm{rad}$ on joint 3 . The maximum value of the SR-6iA is $167.2336 \mathrm{~mm}$ on joint 3 and the minimum is $0.0000 \mathrm{rad}$ on joint 4 .

Table $\mathrm{X}$ shows the joint velocity vs. time of the spline graphs for the three robots. Joint velocity is the derivative of the angle of the joint relative to the robots' zero position. The time is measured in seconds. The maximum value of the $200 \mathrm{iD}$ is $1.7390 \mathrm{rad} / \mathrm{s}$ on joint 3 and minimum is $2.1331 \mathrm{rad} / \mathrm{s}$ on joint 3 . The maximum value of the $200 \mathrm{iC}$ is $1.4772 \mathrm{rad} / \mathrm{s}$ on joint 2 and minimum is $-2.9003 \mathrm{rad} / \mathrm{s}$ on joint 3. The maximum value of the SR-6iA is 621.6667 $\mathrm{mm} / \mathrm{s}$ on joint 3 and the minimum is $-726.3067 \mathrm{~mm} / \mathrm{s}$ on joint 3 .

TABLE X

Spline Path Joint Velocity vs. Time

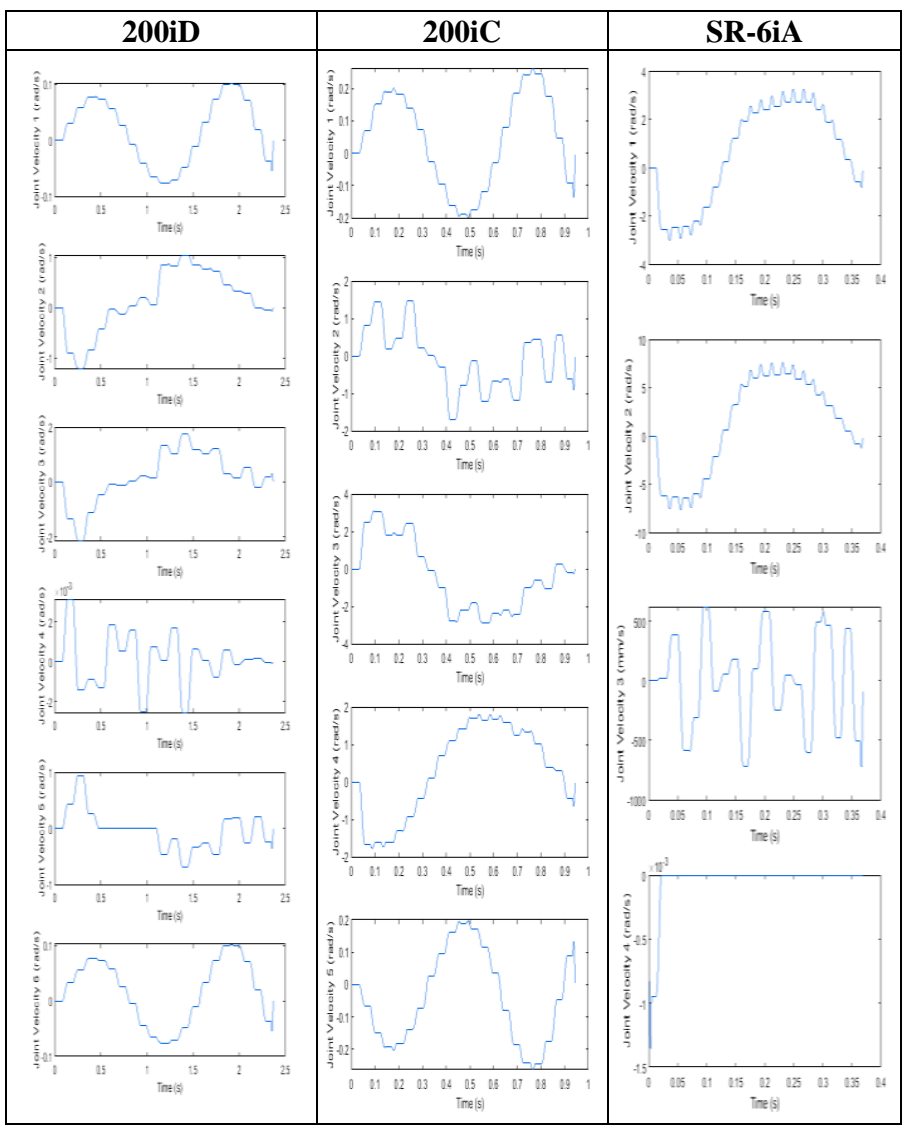

The phase plots in Table XI, shows the joint position vs. joint velocity of the spline graphs for the three robots.

Table XII shows the joint acceleration vs. time of the spline graphs for the three robots. Joint acceleration is the derivative of the joint velocity with respect to time. The time is measured in seconds. The maximum value of the $200 \mathrm{iD}$ is $0.0328 \mathrm{rad} / \mathrm{s}^{2}$ on joint 5 and minimum is -0.0352 $\mathrm{rad} / \mathrm{s}^{2}$ on joint 3 . The maximum value of the $200 \mathrm{iC}$ is $0.1982 \mathrm{rad} / \mathrm{s}^{2}$ on joint 2 and minimum is $-0.1233 \mathrm{rad} / \mathrm{s}^{2}$ on joint 3. The maximum value of the SR-6iA is 349.7333 $\mathrm{mm} / \mathrm{s}^{2}$ on joint 3 and the minimum is $-162.6667 \mathrm{~mm} / \mathrm{s}^{2}$ on joint 3 .

TABLE XI

Spline Path Joint Position vs Joint Velocity

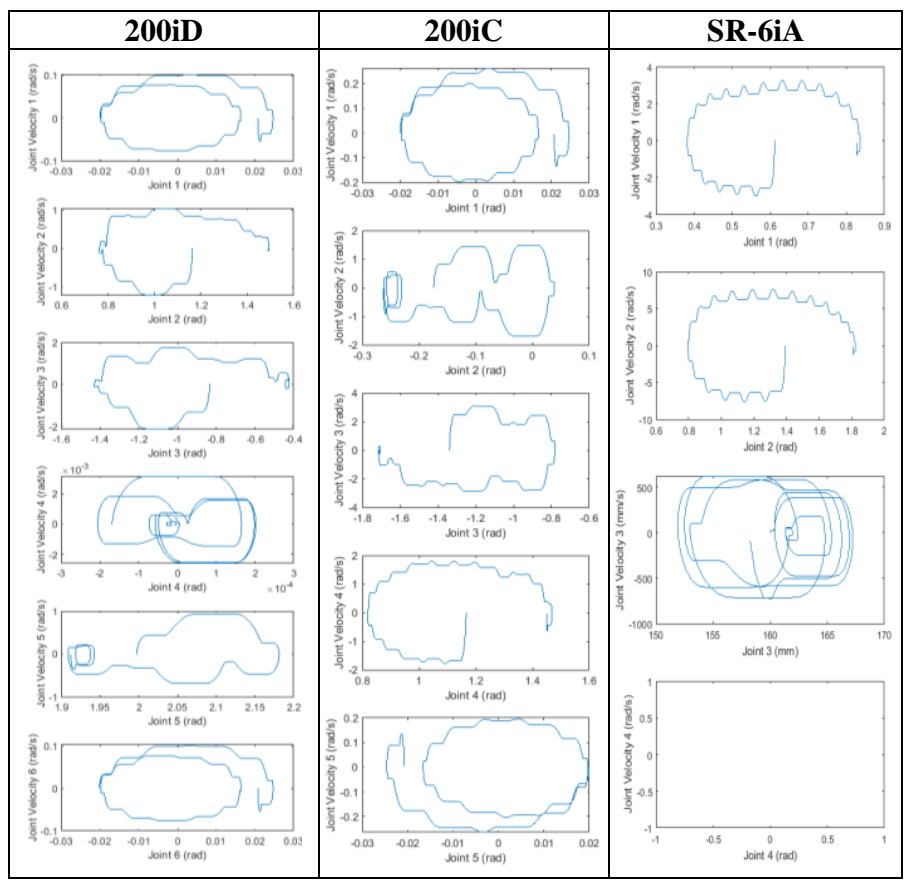




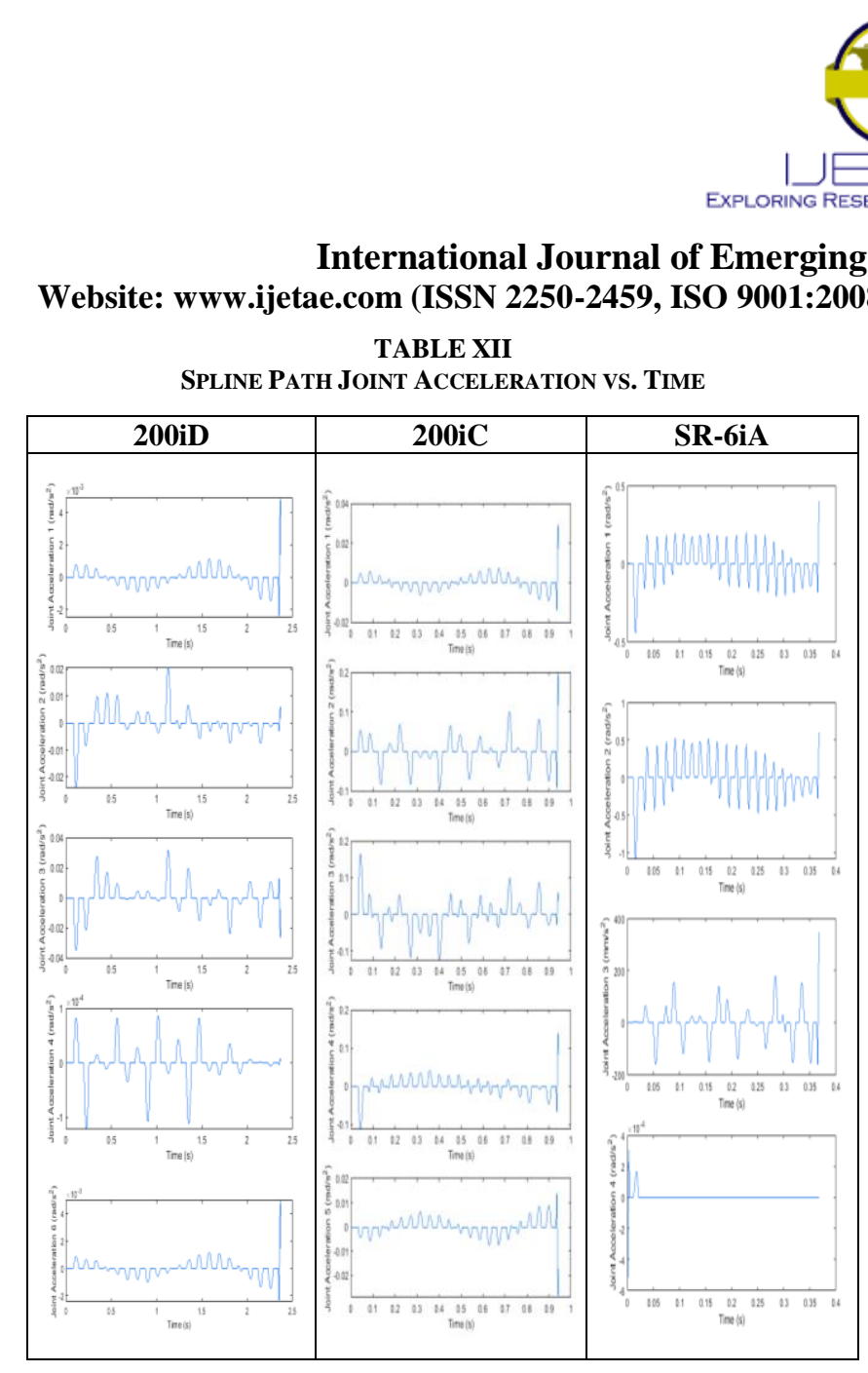

TABLE XIII

Spline Path Cartesian Position vs Time

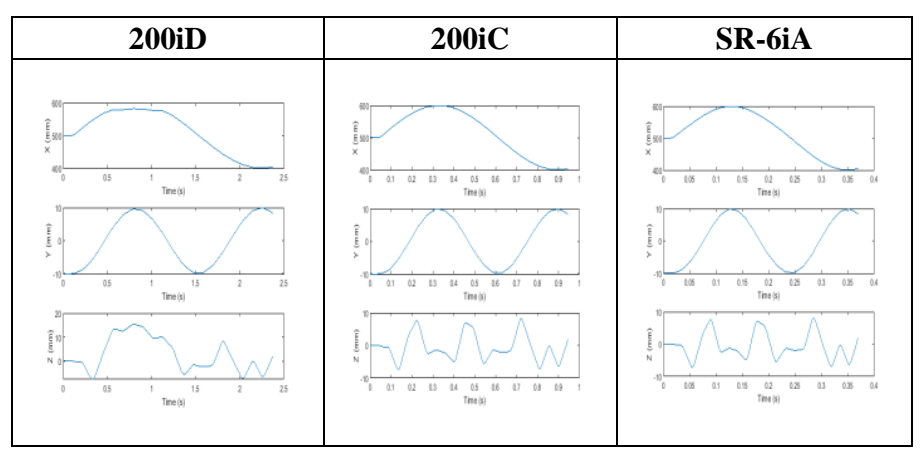

The plots in Table XIII shows the cartesian position vs. time of the spline graphs for the three robots. The 200iD errors in the $\mathrm{X}$ and $\mathrm{Z}$ axes from 0.5 to 1.0 seconds. The $200 \mathrm{iC}$ and SR-6iA both accurately follow the path.

TABLE XIV

Circle Path Joint Position vs. Time

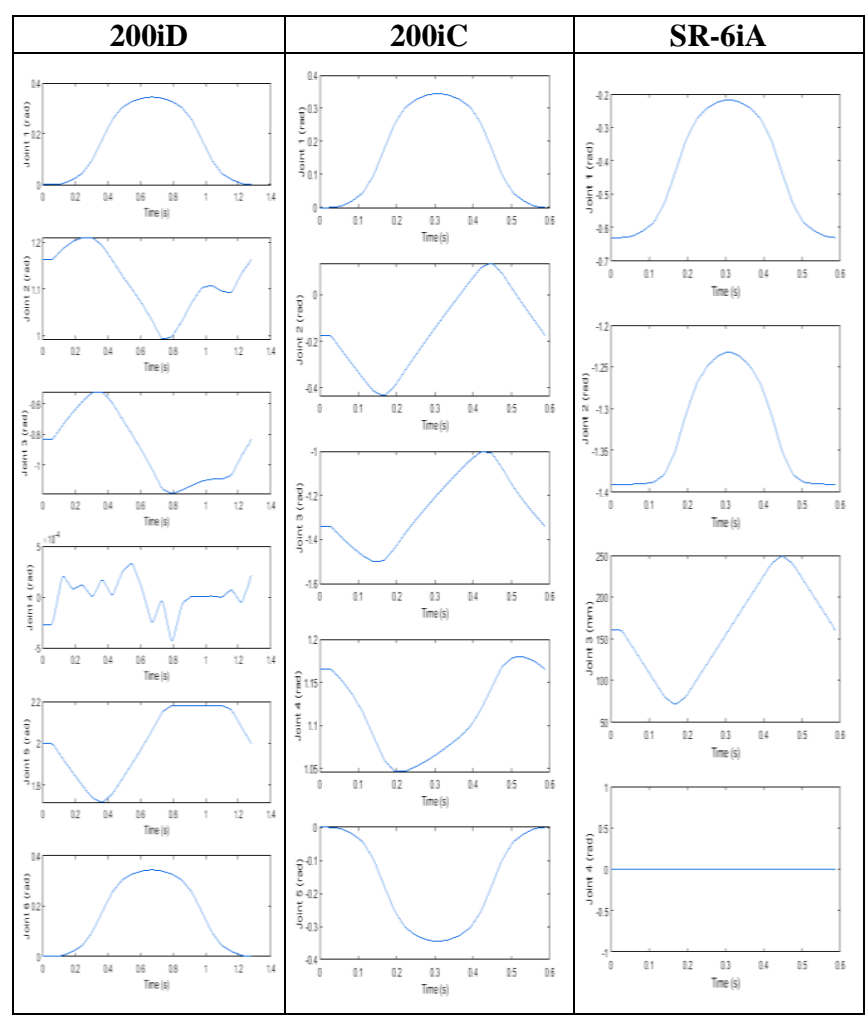

The plots in Table XIV, shows the joint position vs. time of the circle graphs for the three robots. The time is measured in seconds. The maximum value of the 200iD is $2.1817 \mathrm{rad}$ on joint 5 and minimum is $-1.1879 \mathrm{rad}$ on joint 3 . The maximum value of the $200 \mathrm{iC}$ is $1.1794 \mathrm{rad}$ on joint 4 and minimum is -1.5008 on joint 3 . The maximum value of the SR-6iA is $249.0625 \mathrm{~mm}$ on joint 3 and the minimum is $-1.3913 \mathrm{rad}$ on joint 2 . 


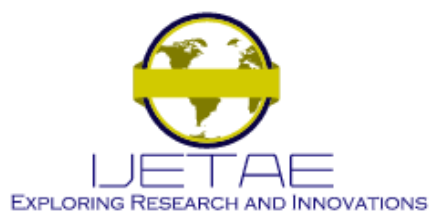

International Journal of Emerging Technology and Advanced Engineering Website: www.ijetae.com (ISSN 2250-2459, ISO 9001:2008 Certified Journal, Volume 10, Issue 11, November 2020

TABLE XV

Circle Path Joint Velocity vs. Time

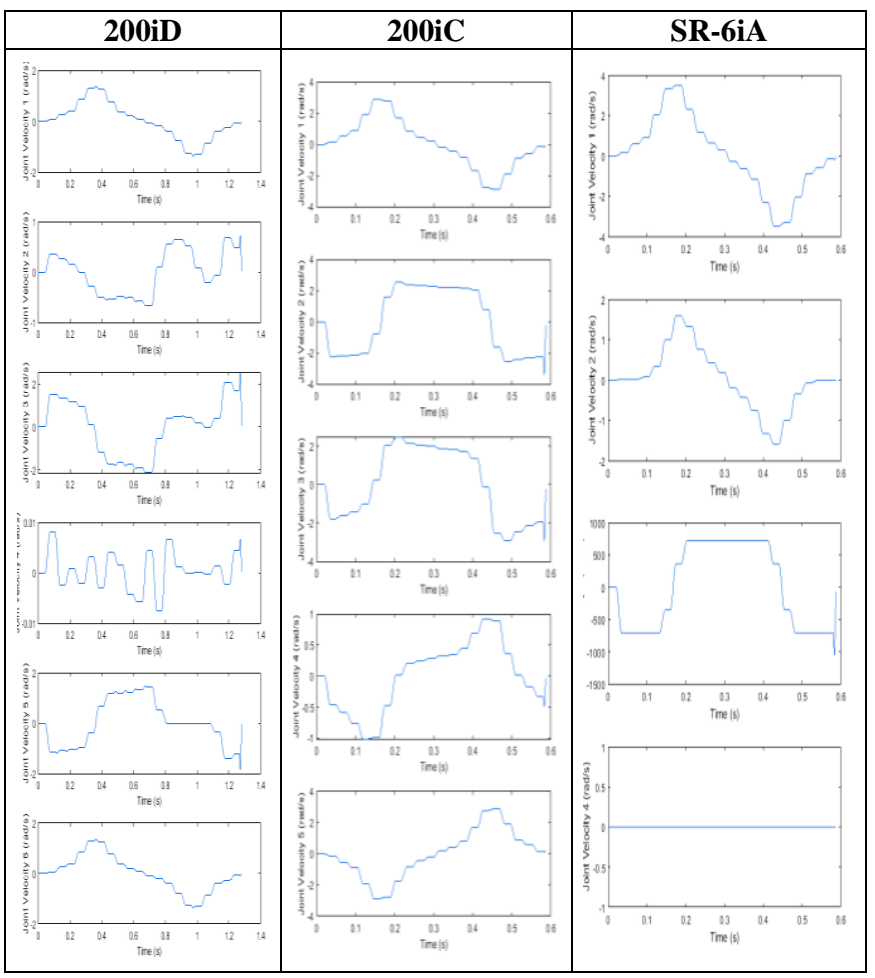

The plots in Table XV shows the joint velocity vs. time of the circle graphs for the three robots. Joint velocity is the derivative of joint position with respect to time. The time is measured in seconds. The maximum value of the $200 \mathrm{iD}$ is $2.1817 \mathrm{rad}$ on joint 5 and the minimum is $-1.1879 \mathrm{rad}$ on joint 3 . The maximum value of the $200 \mathrm{iC}$ is $2.8769 \mathrm{rad} / \mathrm{s}$ on joint 1 and minimum is -3.3861 on joint 2. The maximum value of the SR-6iA is $714.2857 \mathrm{~mm} / \mathrm{s}$ on joint 3 and the minimum is $-1062.9500 \mathrm{~mm} / \mathrm{s}$ on joint 3 .

The plots in Table XVI shows the joint acceleration vs. time of the circle graphs for the three robots. Joint acceleration is the derivative of joint velocity with respect to time.
The time is measured in seconds. The maximum value of the $200 \mathrm{iD}$ is $0.2977 \mathrm{rad} / \mathrm{s}^{2}$ on joint 5 and minimum is $0.4145 \mathrm{rad} / \mathrm{s}^{2}$ on joint 3 . The maximum value of the $200 \mathrm{iC}$ is $1.2017 \mathrm{rad} / \mathrm{s}^{2}$ on joint 2 and minimum is -0.5497 on joint 2. The maximum value of the SR-6iA is $377.2238 \mathrm{rad} / \mathrm{s}^{2}$ on joint 3 and the minimum is $-172.5472 \mathrm{rad} / \mathrm{s}^{2}$ on joint 3 .

TABLE XVI

Circle Path Joint ACCeleration vs. Time

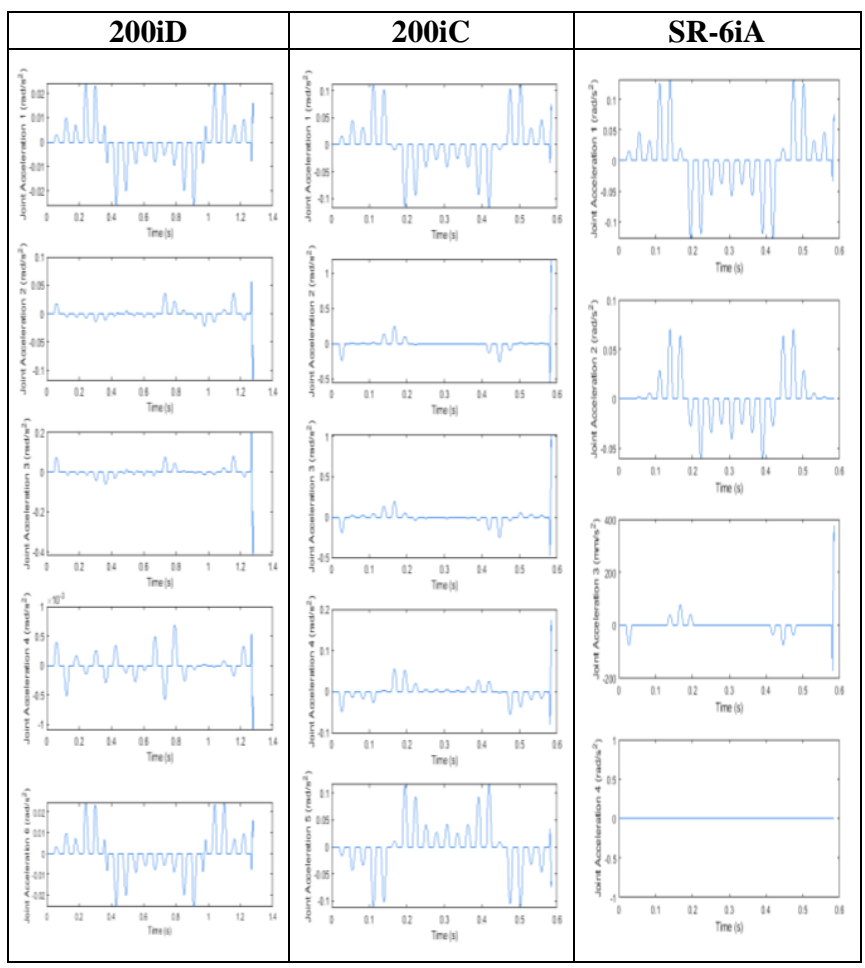

The phase plots in Table XVII shows the joint position vs. joint velocity of the circle graphs for the three robots.

The plot in Table XVIII shows the cartesian position vs. time of the spline graphs for the three robots. 200iC and SR-6iA have a slight $+0.5 /-0.1 \mathrm{~mm}$ variance in the $\mathrm{X}$ axis. The $200 \mathrm{iD}$ errors $16 \mathrm{~mm}$ from 0.8 and 1.1 seconds. 


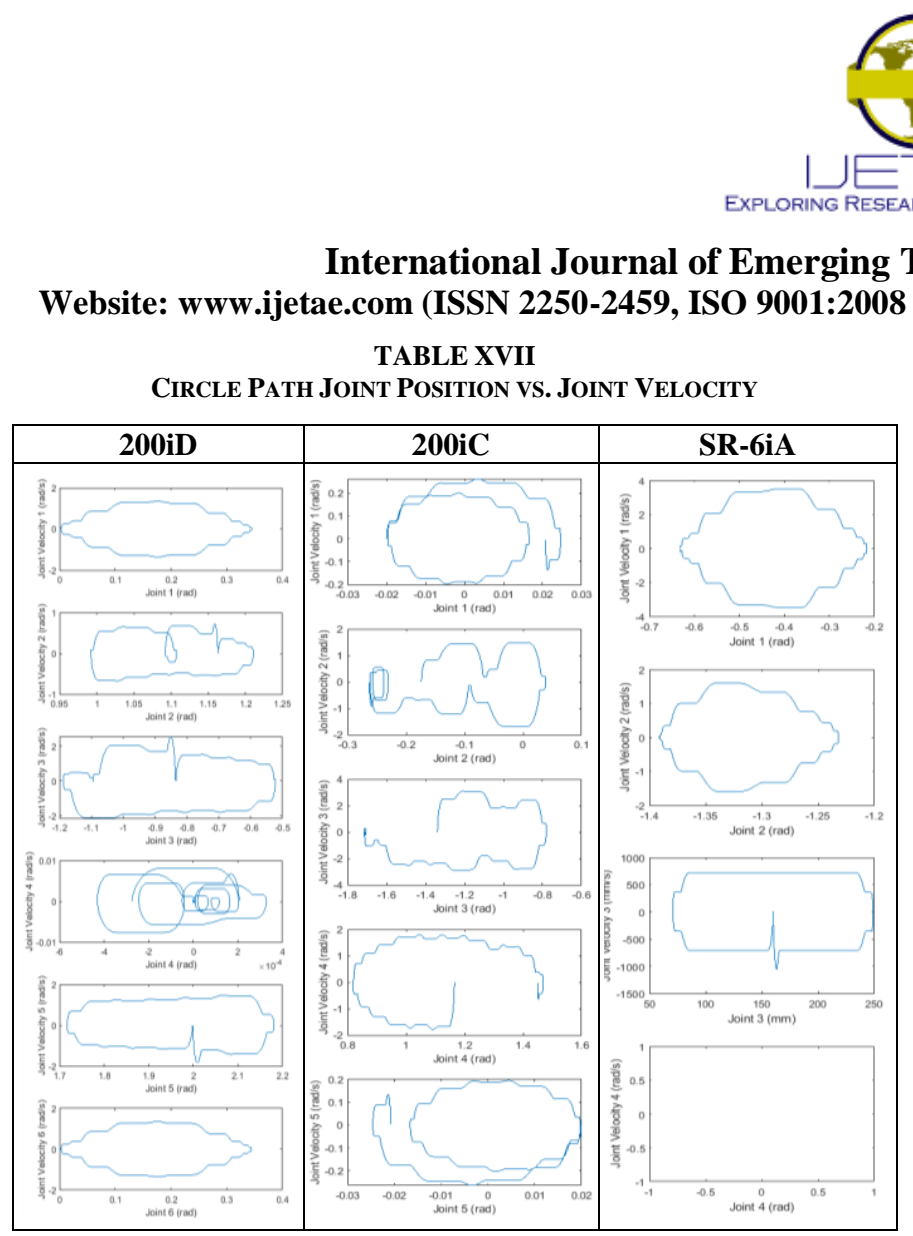

TABLE XVIII

Circle Path Cartesian Position vs Time

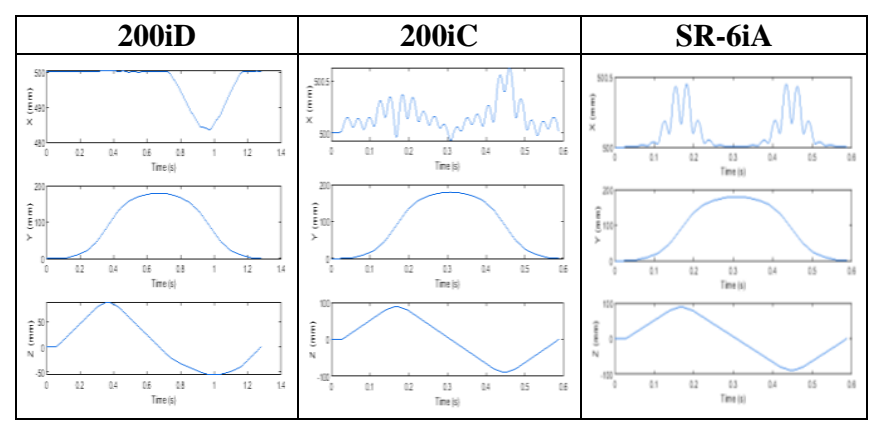

The plots in Table XIX shows the joint position vs. time of the square graphs for the three robots. The time is measured in seconds. The maximum value of the 200iD is $2.1817 \mathrm{rad}$ on joint 5 and the minimum is $-1.4520 \mathrm{rad}$ on joint 3 . The maximum value of the $200 \mathrm{iC}$ is $1.1657 \mathrm{rad}$ on joint 4 and minimum is $-1.3400 \mathrm{rad}$ on joint 3 . The maximum value of the SR-6iA is $160.0000 \mathrm{~mm}$ on joint 3 and the minimum is $-1.3913 \mathrm{rad}$ on joint 2 .

The plots in Table XX shows the cartesian position vs. time of the spline graphs for the three robots. 200iC has slight $\pm 0.2 \mathrm{~mm}$ variance in the $\mathrm{Z}$ axis. The 200iD errors $20 \mathrm{~mm}$ from 0.5 to 1.4 seconds. 


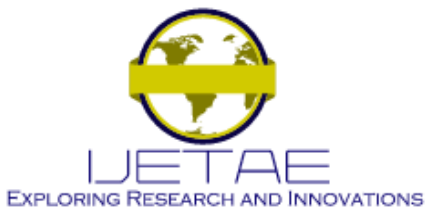

International Journal of Emerging Technology and Advanced Engineering

Website: www.ijetae.com (ISSN 2250-2459, ISO 9001:2008 Certified Journal, Volume 10, Issue 11, November 2020

TABLE XXI

SQuARe PaTh Joint Velocity VS Time

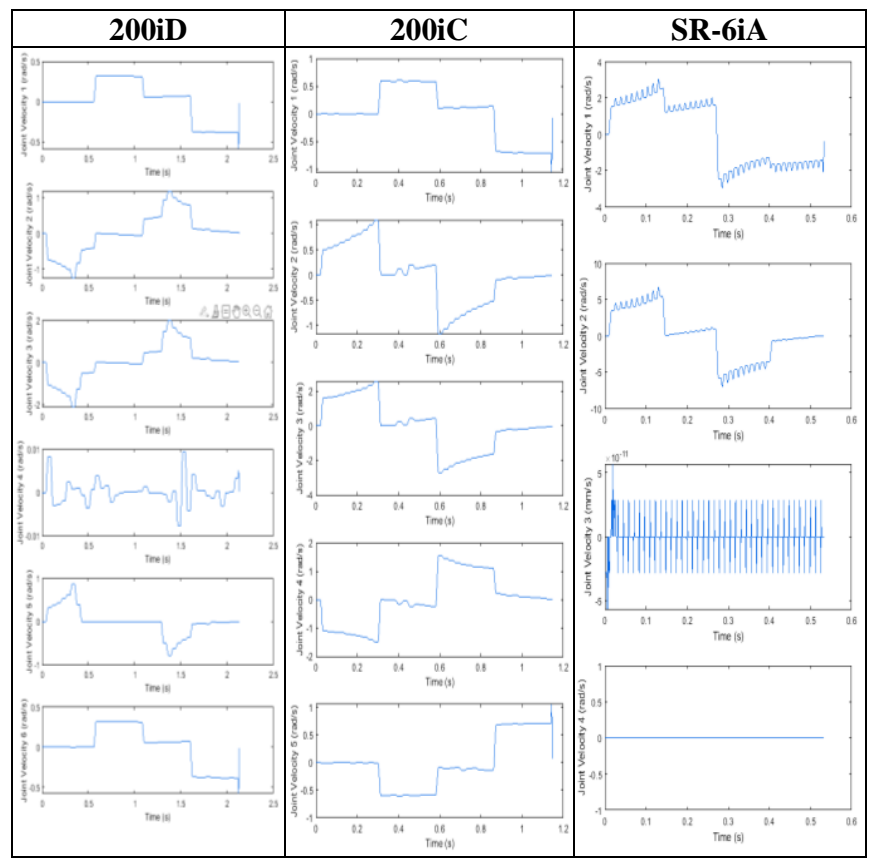

TABLE XXII

SQuare Path Joint Position vs JoInt Velocity

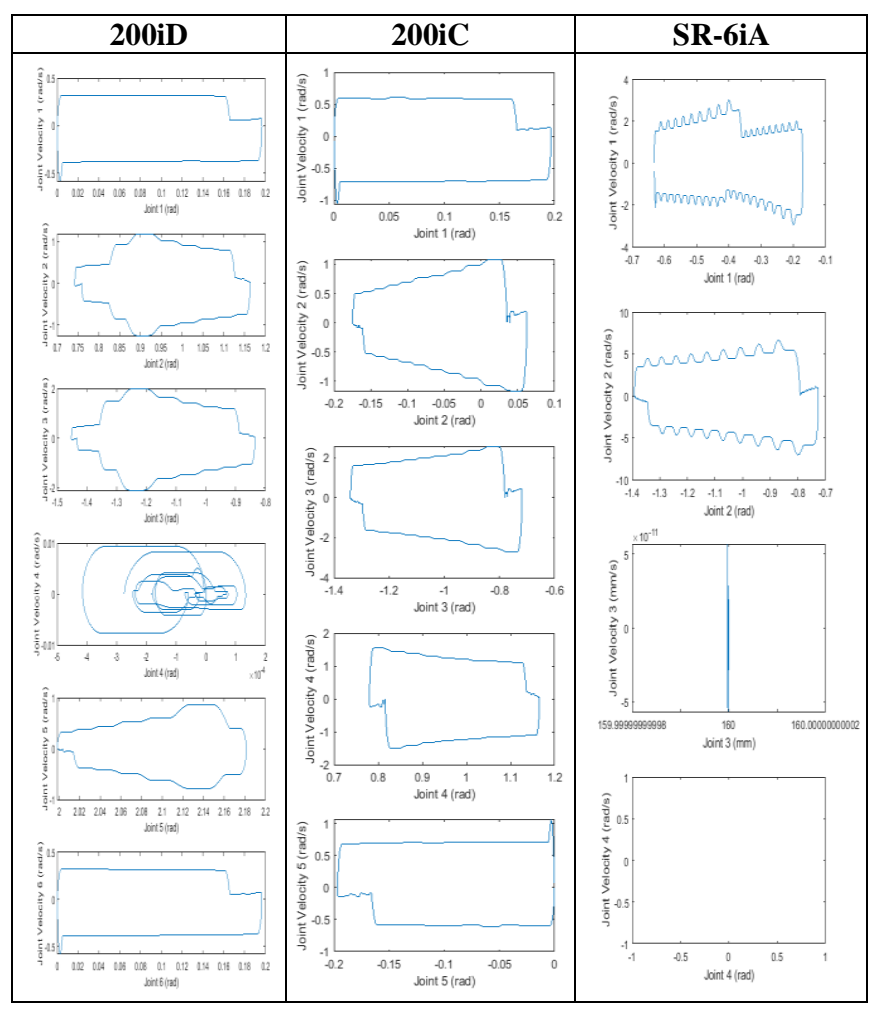

The phase plots in Table XXII shows the joint position vs. joint velocity of the square graphs for the three robots.

TABLE XXIII

SQUARE PATH JOINT ACCELERATION VS TIME

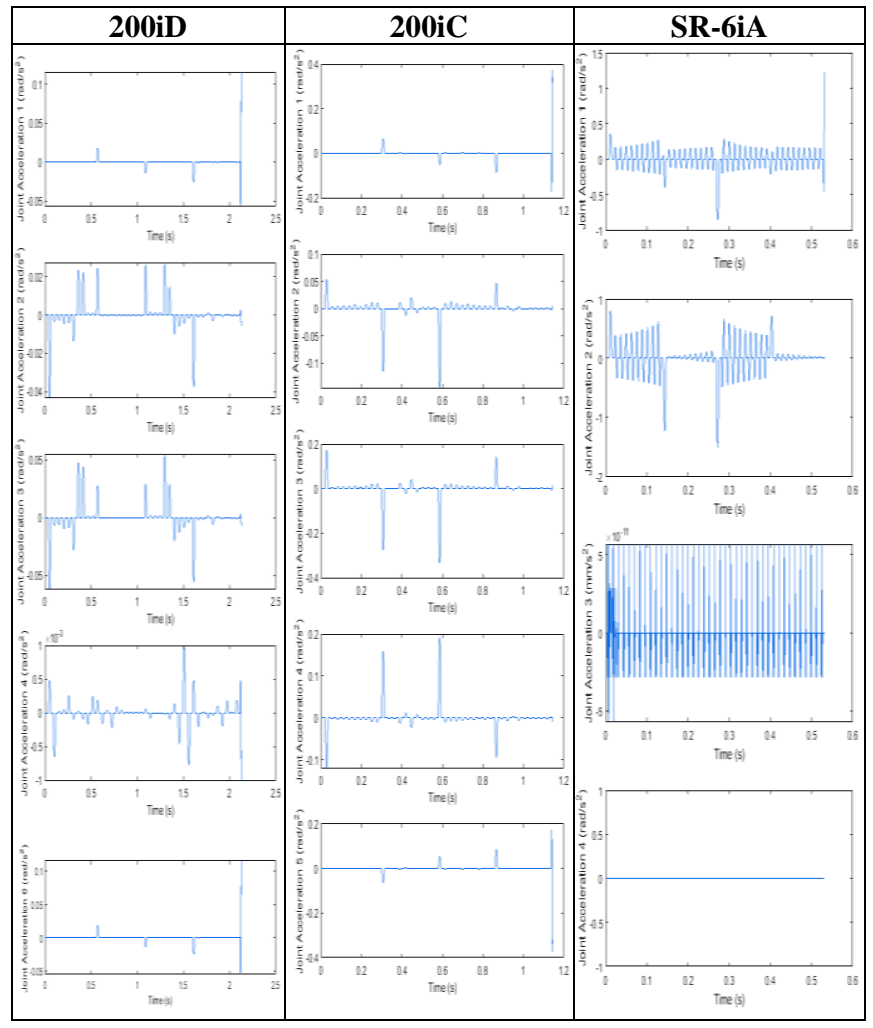

The plots in Table XXIII shows the joint acceleration vs. time of the square graphs for the three robots. The time is measured in seconds. The maximum value of the $200 \mathrm{iD}$ is $0.1157 \mathrm{rad} / \mathrm{s}^{2}$ on joint 1 and the minimum is $-0.0614 \mathrm{rad} / \mathrm{s}^{2}$ on joint 3. The maximum value of the $200 \mathrm{iC}$ is 0.3755 $\mathrm{rad} / \mathrm{s}^{2}$ on joint 1 and minimum is $-0.3755 \mathrm{rad} / \mathrm{s}^{2}$ on joint 5 . The maximum value of the SR-6iA is $1.2292 \mathrm{rad} / \mathrm{s}^{2}$ on joint 1 and the minimum is $-1.5128 \mathrm{rad} / \mathrm{s}^{2}$ on joint 2 .

\section{RESULTS ANALYSIS}

The position, velocity, and acceleration vs. time plots show the trajectory curves of each joint. The curves take into consideration the maximum joint velocity and joint limits. 


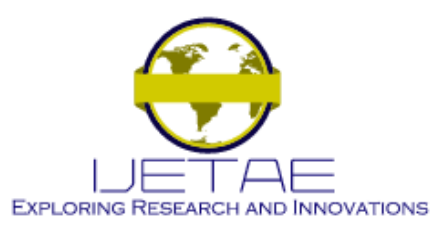

\section{International Journal of Emerging Technology and Advanced Engineering Website: www.ijetae.com (ISSN 2250-2459, ISO 9001:2008 Certified Journal, Volume 10, Issue 11, November 2020}

A phase space plot shows the relation of the joint position to the joint velocity. Since no point on any of paths operates at a speed greater than the maximum joint velocity and the time acceleration is set to the minimum, the phase plots show the optimal trajectory curves to complete the paths. As joint 4 of the SR-6iA does not move in any curve, each phase plot of joint 4 is an equilibrium point.

Overall, the SR-6iA is notably faster than the $200 \mathrm{iC} / 5 \mathrm{H}$ and the 200iD in all cases. The average speed of the robots are 1.9293 seconds for the $200 \mathrm{iD}, 0.8940$ seconds for the $200 \mathrm{iC} / 5 \mathrm{H}$, and 0.4940 seconds for the SR-6iA. The SR-6iA is $80.97 \%$ faster on average than the $200 \mathrm{iC} / 5 \mathrm{H}$ and the $200 \mathrm{iC} / 5 \mathrm{H}$ is $115.81 \%$ faster than the $200 \mathrm{iD}$ on average. The Sr-6iA's speed advantage mostly affects paths which have minimal Z-axis movement. The SR-6iA is $114.98 \%$ faster than the $200 \mathrm{iC} / 5 \mathrm{H}$ on the square path where movement is only along the $\mathrm{X}-\mathrm{Y}$ plane, but completes the circle path at the exact same time which is along the Y-Z.

\section{CONCLUSION}

The forward and inverse kinematics of the Fanuc $200 \mathrm{iC} / 5 \mathrm{H}, 200 \mathrm{iD}$, and SR-6iA were clarified and calculated from the information found in the paper, Efficiency Comparison Between Robotic Manipulator Configurations for Trajectory/Path Tracing. Three paths were defined for the robots to follow. A MATLAB program was written to compute the trajectory of the robots at maximum speed for the given paths with respect to the joint and velocity limits and plot the joint position, velocity, and acceleration vs time graphs as well as cartesian position vs time. The SCARA robotic arm was found to be considerably quicker in completing paths compared to the articulated arms and the lower degree of freedom arms were quicker than the high degree of freedom arms. The SCARA configuration was found most advantageous in paths with minimal $\mathrm{Z}$-axis travel.

\section{RECOMMENDATION}

To expand on the analysis of these robot arms, a future study should compare results with the actual robots or try different trajectory generation such as genetic algorithms and fuzzy logic.
This paper was also only concerned with the kinematics of the robot and does not consider the dynamics. A future study should consider the dynamics with payload.

\section{REFERENCES}

[1] Laber, J., and Thamma, R. "Efficiency Comparison Between Robotic Manipulator Configurations for Trajectory/Path Tracing," International Journal of Emerging Technology and Advanced Engineering, ISSN: 2250-245 Volume 7. no. 11 (November 2020).

[2] Hauser, Kris. "Section II. MODELING" Robotic Systems (draft), http://motion.cs.illinois.edu/RoboticSystems/Kinematics.html. Accessed 12 Nov. 2020

[3] Lynch, Kevin, and Frank C. Park. Modern robotics: mechanics, planning, and control. Cambridge, United Kingdom New York, NY: Cambridge University Press, 2017. Print.

[4] J. Denavit, and R. Hartenberg: ASME Journal of Applied Mechnics Vol. 22(1955), No. 6, p. 215.

[5] Spong, Mark W, et al. Robot Dynamics and Control 2nd ed., Wiley, 2004.

[6] Muhammed Enes Uk; Faris Bin Sajjad Ali Shah; Mucahit Soyaslan; Osman Eldogan. "Modeling, control, and simulation of a SCARA PRR-type robot manipulator". Scientia Iranica, 27, 1, 2020, 330-340. doi: $10.24200 /$ sci.2018.51214.2065

[7] Soyaslan, M. et al. "Modelling, Control and Simulation of a SCARA PRR-Type Robot Manipulator.” Scientia Iranica 27 (2018): 330-340.

[8] P.I. Corke, Robotics, Vision \& Control: Fundamental Algorithms in MAT-LAB. Second edition. Springer, 2017. ISBN 978-3-319$54413-$

[9] Bobrow, J. E., Dubowsky, S., \& Gibson, J. S. (1985). Time-Optimal Control of Robotic Manipulators Along Specified Paths. The International Journal of Robotics Research, 4(3), 3-17. doi:10.1177/027836498500400301

[10] Gustafson, G.B. "Chapter 10" Phase Plane Methods, https://www.math.utah.edu/ gustafso/f2010/dynamicalSystems.pdf. Accessed 25 Nov. 2020.

[11] Holgate, M. A., Sugar, T. G., \& Bohler, A. W. (2009). A novel control algorithm for wearable robotics using phase plane invariants. 2009 IEEE International Conference on Robotics and Automation. doi:10.1109/robot.2009.5152565

[12] "About" Peter Corke, petercorke.com/about/. Accessed 26 Nov. 2020.

[13] "Robotics Toolbox" Peter Corke, petercorke.com/toolboxes/robotics-toolbox/. Accessed 26 Nov. 2020. 